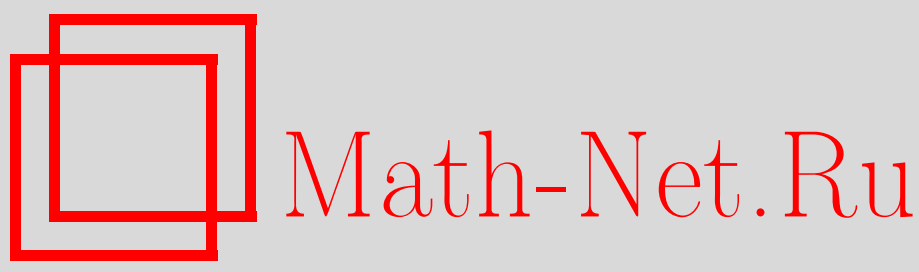

О. В. Дружинина, А. А. Шестаков, Обобщенный прямой метод Ляпунова исследования устойчивости и притяжения в общих временны́х системах, Матем. сб., 2002, том 193, номер 10, 17-48

DOI: https://doi.org/10.4213/sm684

Использование Общероссийского математического портала Math-Net.Ru подразумевает, что вы прочитали и согласны с пользовательским соглашением

http://www.mathnet.ru/rus/agreement

Параметры загрузки:

IP: 3.85 .5 .30

26 апреля 2023 г., 17:58:23 


\title{
Обобщенный прямой метод Ляпунова исследования устойчивости и притяжения в общих временнь́х системах
}

\begin{abstract}
В настоящей статье развит обобщенный прямой метод Ляпунова исследования устойчивости и притяжения в общих временнь́х системах следующих типов: в классической динамической системе в смысле Биркгофа, в общей системе в смысле Зубова, в общей системе в смысле Сейберта, в общей системе с запаздыванием и в общей системе "вход-выход". Для указанных систем с помощью обобщенных функций Ляпунова относительно двух фильтров, двух квазифильтров или двух базисов фильтров получены необходимые и достаточные условия устойчивости и притяжения при минималњных предположениях на математическую структуру общей системы.

Библиографоия: 40 названий.
\end{abstract}

\section{$\S 1$. Введение}

В работах А. М. Ллпунова [1], А. Пуанкаре [2] заложены основы метода изучения свойств устойчивости и притяжения решений обыкновенных дифференциальных уравнений, называемого прямым методом Ляпунова, или методом функuий Ляпунова. В дальнейшем прямой метод Ляпунова был сушественно развит в самых различных направлениях в многочисленных трудах отечественных и зарубежных ученых и получил название обобщенного прямого метода Ляпунова, или метода обобщенных функций Ляпунова (см., например, монографии [3]-[9], а также обзор [10]).

Обобщенный прямой метод Ляпунова стал одним из основных методов исследования динамических и качественных свойств решений различњых типов эволюционных уравнений. Крупные достижения в развитии обобщенного метода Ляпунова принадлежат Н.Г. Четаеву [3], В.В. Румянщеву [11], [10], К. П. Персидскому [12], Н.Н. Красовскому [13], В.И. Зубову [14], В.М. Матросову [15] и другим ученым, предложившим обобшения и модификации базовых теорем Ллпунова с ослаблением многих требований к классическим функциям Ляпунова. Указанные исследования расширили классы функций Ляпунова для решения задач устойчивости. Область применимости обобщенного прямого метода Ляпунова была распространена и на счетные и другие бесконечные системы дифференциальных уравнений (К.П. Персидский [12]), на дифференциальные уравнения в частных производных (В.И. Зубов [14], А.В. Бабин и М.И. Вишик [7], А. А. Мовчан [16]). В работах Е. А. Барбашина [17], В. И. Зубова [14], А. А. Мовчана [16] и других ученых метод обобшенных функций Ляпунова распространен на общие системы в метрическом и

Работа вьполнена при поддержке Министерства образования РФ (грант № 020702-2-075).

(C) О. В. ДРУжинина, А. А. ШестАков 2002 
топологическом пространствах, в пространстве сходимости Фреше, в множестве, наделенном некоторой структурой предпорядка.

Для общих систем, охватываюших динамические системы и другие типы систем, к настояшему времени предложено большое число различных понятий устойчивости и притяжения и соответственно понятий обобщенной функции Ляпунова. В.И. Зубов [14] ввел понятие общей системы на произвольных метрических пространствах и исследовал устойчивость и притяжение в этих системах с помощью обобщенных функций Ляпунова. Д. Башо [18] предложил определение устойчивости в полисистемах и установил критерий устойчивости с помощњю обобщенных функций Ляпунова.

А.А. Мовчан [16] рассмотрел устойчивость по двум метрикам в общих системах с двумя метриками. В.В. Немыцкий и В.В. Степанов [19], [20] изучили ряд свойств устойчивости в общих динамических системах. В [21] С. Ёсии установил необходимые и достаточные условия устойчивости в системах "вход-выход".

В [7]-[9], [22]-[24] представлены суммирующие результаты по обобщенному прямому методу Ляпунова исследования динамических и качественных свойств решений для систем с распределенными параметрами. В [24] предложено понятие абстрактного эволюционного уравнения, охватывающее нечеткие дифференциальные уравнения, стохастические дифференциальные уравнения и дифференциальные включения, и изучены динамические свойства решений с помощью обобщенных функций Ляпунова.

В настоящей работе на базе обобшенного прямого метода Ляпунова предложен универсальньй подход к исследованию устойчивости и притяжения в общих системах на пространствах, наделенных той или иной математической структурой, в частности в классических динамических системах, в общих системах с заданњым предпорядком, в обших системах “вход-выход”. В работе рассмотрены такие виды устойчивости общей системы, как устойчивость в смысле Ляпунова, орбитальная устойчивость отдельной траектории и множества траекторий, устойчивость реакции систем “вход-выход”, устойчивость при постоянно действующих возмущениях, устойчивость в смысле Пуассона, устойчивость в смысле Немыцкого-Степанова. В статье продолжены исследования авторов [25]-[28].

Приведем краткое содержание работы. В $\S 2$ даны сведения из теории обобшенных функций Ляпунова для общих систем на множествах, наделенных той или иной математической структурой. В $\S 3$ изучена устойчивость множества в классической динамической системе с помощью обобщенных функций Ляпунова относительно двух фильтров. В $\S \S 4$ и 5 исследована устойчивость структуры предпорядка в общей системе с заданным предпорядком с помощью обобщенных функций Ляпунова относительно двух квазифильтров и двух базисов фильтров соответственно. В $\S 6$ доказаны теоремы об устойчивости и асимптотической устойчивости решения в смысле Ляпунова в общей системе с запаздыванием с помощью обобщенных функций Ляпунова относительно двух метрик. В завершающем $\S 7$ исследованы различные виды устойчивости в общей системе "вход-выход" с помощью обобшенных функций Ляпунова. Здесь основное структурное свойство обобщенных функций Ляпунова положено в основу определения понятия устойчивости. Это структурное свойство позволило установить критерий устойчивости для общей системы, обладающей достаточно слабой математической структурой фазового пространства. Этот критерий (названньй ключевым) непосредственно переносится на лю- 
бую общую систему с более сильной математической структурой фазового пространства, такой, которая не нарушает условий критерия.

В дальнейшем будем использовать следующие обозначения:

1) $\mathbb{R}=(-\infty,+\infty), \mathbb{R}^{+}=[0, \infty), \mathbb{R}^{-}=(-\infty, 0]$;

2) $\mathbb{R}^{n}-n$-мерное евклидово пространство;

3) $A, B$ - произвольные абстрактные множества;

4) $S(A)$ - совокупность всех непустых подмножеств множества $A$;

5) $B(M, \rho)$ - шар радиуса $\rho$ с центром в $M$;

6) $C(x), C^{+}(x), C^{-}(x)$ - траектория, положительная и отрицательная полутраектории, проходящие через $x \in A$;

7) $C(U), C^{+}(U), C^{-}(U)$ - совокупность всех траекторий, всех положительных и отрицательных полутраекторий, проходящих через точки множества $U \subset A$;

8) $T$-множество моментов времени с определенньм на нем отношением порядка $\leqslant$;

9) $A^{T}$ и $B^{T}$ - множества всевозможных отображений из $T$ в $A$ и $B$ соответственно;

10) $X \subset A^{T}, Y \subset B^{T}$ - множества, элементы которых суть абстрактные функции времени;

11) $x: T \rightarrow A$ и $y: T \rightarrow A$; $x(t)$ и $y(t)$ - значения функций из $X$ и $Y$ в момент $t$;

12) для любых $t \quad T_{t}=\left\{t_{1}: t_{1} \geqslant t\right\}, T^{t}=\left\{t_{1}: t_{1}<t\right\}, \bar{T}^{t}=T^{t} \cup\{t\}$; $T_{t t_{1}}=\left\{\tau: t \leqslant \tau<t_{1}\right\}, \bar{T}_{t \tau}=T_{t \tau} \cup\left\{t_{1}\right\}$

13) $x_{t}=x\left|T_{t}, \quad x^{t}=x\right| T^{t}, \quad x_{t \tau}=x\left|T_{t \tau}, \quad \bar{x}_{t \tau}=x\right| \bar{T}_{t \tau}, \bar{x}^{t}=x \mid \bar{T}^{t}$, $X_{t}=\left\{x_{t}: x_{t}=x \mid T_{t} \wedge x \in X\right\}, X^{t}=\left\{x^{t}: x^{t}=x \mid T^{t} \wedge x \in X\right\}$, $X_{t \tau}=\left\{x_{t \tau}: x_{t \tau}=x \mid T_{t \tau} \wedge x \in X\right\}-$ сужения функции $x \in A^{T}$ на различных отрезках времени;

14) $X(t)=\{x(t): x \in X\}, x_{t t}=\varnothing, X_{t t}=\{\varnothing\}$.

\section{§2. Некоторые сведения из теории обобщенных функций Ляпунова}

2.1. Обобщенные функции Ляпунова для $(A, f, \mathbb{R})$-системы. Следуя [29] и [20], приведем определение классической динамической системы. Пусть $A-$ метрическое пространство и задано однопараметрическое семейство отображений $f: A \rightarrow A$ пространства $A$ на себя, ставяшее каждой точке $z \in A$ и любому вещественному числу из $\mathbb{R}$ некоторую фиксированную точку $f(t, z) \in A$. На отображение $f: A \rightarrow A$ наложим следуюшие условия:

1) начальное условие: $f(0, z)=z$ для всех $z \in A$;

2) условие непрерьвности по совокупности $(t, z) \in \mathbb{R} \times A$;

3) условие группы: $f\left(t_{2}, f\left(t_{1}, z\right)\right)=f\left(t_{1}+t_{2}, z\right)$ для любых действительных чисел $t_{1}$ и $t_{2}$ и для каждого $z \in A$.

В силу условия 1 ) значению $t=0$ соответствует тождественное преобразование групшы. Из условий 1) и 3 ) вытекает сушествование преобразования $f(-t, z)$, обратного к преобразованию $f(t, z)$, так как $f(t, f(-t, z))=z$.

Тройка $(A, f, \mathbb{R})$ называется классической динамической системой на пространстве $A$ (или для краткости $(A, f, \mathbb{R})$-системой), если $f(t, z)$ есть группа преобразований пространства $A$ на себя, обладаюшая вьшеприведенными свойствами 1)-3). 
ЗАмечАниЕ. В математической литературе $(A, f, \mathbb{R})$-система называется также динамической системой Биркгофа, динамической системой Немњцкого-Степанова или автономньм динамическим процессом.

Напомним некоторые понятия. Функция $f(t, z)$ при фиксированном $z$ называется движением, а множество точек $\{f(t, z): t \in \mathbb{R}\}$ назьвается траекторией $C(z)$ этого движения. Множества $\left\{f(t, z): t \in \mathbb{R}^{+}\right\}$и $\left\{f(t, z): t \in \mathbb{R}^{-}\right\}$соответственно называются положительной и отрицательной полутраекториями и обозначаются через $C^{+}(z)$ и $C^{-}(z)$.

Точка $z \in A$, для которой $f(t, z)=z$ при каждом $t \in \mathbb{R}$, называется состоянием равновесия (точкой покоя) $(A, f, \mathbb{R})$-системы. Периодическим движением с периодом $\tau$ назьвается такое движение $f(t, z)$, для которого $f(t+\tau, z)=f(t, z)$ при каждом $t \in \mathbb{R}$. Непериодическим движсением называется такое движение $f(t, z)$, для которого $f\left(t_{1}, z\right) \neq f\left(t_{2}, z\right)$ при $t_{1} \neq t_{2}$.

Имеются три существенно различных топологических типа траекторий $(A, f, \mathbb{R})$-системы: 1) точка, 2) простая замкнутая кривая, 3) взаимно однозначный и непрерывный образ открытого интервала. Этим типам траекторий соответствуют три типа движений: 1) состояние равновесия, 2) периодическое движение, 3) непериодическое движение.

Образ множества $M \subset A$ при преобразовании группы для фиксированного $t$ обозначим через $f(t, M)$. Множество $M \subset A$ называется инвариантным (соответственно положстельно инвариантным ), если $f(t, M)=M \forall t \in \mathbb{R}$ (соответственно $\left.f(t, M)=M \forall t \in \mathbb{R}^{+}\right)$.

Легко видеть, что инвариантное множество состоит из целых траекторий.

Пусть $M \subset A$ - компактное положительно инвариантное множество $(A, f, \mathbb{R})$ системы. Множество $M$ называется

1) положительно орбитально устойчивым, если для любого $\varepsilon>0$ сушествует число $\delta>0$ такое, что $f(t, B(M, \delta)) \subset B(M, \varepsilon)$,

2) асимптотически положительно орбитально устойчивым, если оно положительно орбитально устойчиво и существует окрестность $N$ множества $M$ такая, что $\forall x \in N \rho(f(t, x), M) \rightarrow 0, t \rightarrow+\infty$, где $\rho$ - расстояние от точки до множества.

ОПРЕДЕЛЕНИЕ 2.1 [30]. Пусть $M \subset A$ - компактное инвариантное множество. Неотрицательная функция $V: A \rightarrow \mathbb{R}^{+}$называется обобщенной функиией Ляпунова $(A, f, \mathbb{R})$-системь для множества $M$, если она определена на положительно инвариантной окрестности $N$ множества $M$ и удовлетворяет условиям:

1) если $\varepsilon>0$, то сушествует $c>0$ такое, что $V(x)>c \forall x \notin B(M, \varepsilon)$;

2) если $\lambda>0$, то существует $d>0$ такое, что $V(x)<\lambda \forall x \in B(M, d)$;

$3)$ если $x \in N$ и $t \geqslant 0$, то $V(f(t, x)) \leqslant V(x)$.

ПРЕДЛОЖЕНИЕ 2.1 [30]. Компактное множсество $M \subset A(A, f, \mathbb{R})$-системь

1) полохсительно орбитально устойчиво тогда и только тогда, когда существует обобщенная функиия Ляпунова для $M$,

2) асимптотически положстельно орбитально устойчиво тогда и только тогда, когда существует обобщенная непрерьвная функция Ляпунова для множества $M$ такая, что если $x \notin M u t>0$, то $V(f(t, x))<V(x)$. 
2.2. Обобщенные функции Ляпунова для $\left(A, F_{t_{0}}^{t}, \mathbb{R}\right)$-системы. Пусть $A$ - метрическое пространство с метрикой $\rho$. В [14] введено понятие общей системы, которая в дальнейшем была названа [15] классической общей системой. Тройка $\left(A, F_{t_{0}}^{t}, \mathbb{R}\right)$ называется классической общей системой (или для краткости $\left(A, F_{t_{0}}^{t}, \mathbb{R}\right)$-системой), если $F_{t_{0}}^{t}$ представляет собой двупараметрическое семейство операторов, $F_{t_{0}}^{t}(x): A \rightarrow A$, обладаюшее свойствами:

а) для любого $x \in A$ и $t_{0} \geqslant 0$ определено множество $F_{t_{0}}^{t}(x) \subset A \forall t \geqslant t_{0}$ и $F_{t_{0}}^{t}(x)$ непусто;

б) $F_{t_{0}}^{t}(x) \subset A \forall t \geqslant t_{0}$ при $t \rightarrow t_{0}+0$;

в) для любого элемента $x_{1} \in F_{t_{0}}^{t_{1}}$ определено множество $F_{t_{1}}^{t}\left(x_{1}\right)$ такое, что

$$
\bigcup_{x_{1} \in F_{t_{0}}^{t_{1}}(x)} F_{t_{1}}^{t}\left(x_{1}\right)=F_{t_{0}}^{t}(x) \quad \forall t \geqslant t_{1} .
$$

При фиксированном $x$ функция $F_{t_{0}}^{t}(x)$ называется движением $\left(A, F_{t_{0}}^{t}, \mathbb{R}\right)$-системы. Положим

$$
\rho\left(t, x_{0}, t_{0}\right)=\sup _{x \in F_{t_{0}}^{t}\left(x_{0}\right)} \rho(x, M), \quad M \subset A .
$$

Пусть $M \subset A$ - инвариантное множество $\left(A, F_{t_{0}}^{t}, \mathbb{R}\right)$-системы. Множество $M$ называется положительно орбитально устойчивым, если

$$
\forall \varepsilon>0 \quad \exists \delta(\varepsilon)>0 \quad \rho\left(x_{0}, M\right)<\delta \Rightarrow \rho\left(t, x_{0}, t_{0}\right)<\varepsilon \quad \forall t \geqslant t_{0} \geqslant 0 .
$$

Если, кроме того, $\rho\left(t, x_{0}, t_{0}\right) \rightarrow 0$ при $t \rightarrow+\infty$, то множество $M$ назьвается асимптотически положительно орбитально устойчивым.

ОПРеДЕЛЕНИЕ 2.2 [14]. Функция $V: \mathbb{R}^{+} \times B(M, r) \rightarrow \mathbb{R}$ называется обобщенной функцией Ляпунова для $\left(A, F_{t_{0}}^{t}, \mathbb{R}\right)$-системь, если

1) $V(t, x) \geqslant 0 \forall(t, x) \in \mathbb{R}^{+} \times B(M, r)$,

2) $\lim V(t, x) \rightarrow 0$ при $\rho(x, M) \rightarrow 0$ равномерно по $t$,

3) для каждого числа $c_{1} \geqslant 0$ сушествует число $c_{2}>0$ такое, что

$$
\rho(x, M)>c_{1} \Rightarrow V(t, x)>c_{2} \quad \forall t \in \mathbb{R}^{+},
$$

4) $V(t, \tau, x):=\sup _{z \in F_{\tau}^{t}(x)} V(t, z)$ не возрастает при всех $t \geqslant \tau$, при которых она определена.

ПрЕДЛОЖЕНИЕ 2.2 [14]. Существование обобщенной функции Ляпунова $V(t, x): \mathbb{R}^{+} \times B(M, r) \rightarrow \mathbb{R}$ со свойствами 1$\left.)-4\right)$ (соответственно со свойствами 1)-4) и свойством $\left.\exists \delta>0 \lim _{t \rightarrow \infty} V(t, \tau, x)=0 \quad \forall x \in B(M, \delta), \forall \tau \in \mathbb{R}^{+}\right)$ является необходимым и достаточныцм условием положстельной орбитальной устойчивости (соответственно асимптотической положительной орбитальной устойчивости) инвариантного множества $M\left(A, F_{t_{0}}^{t}, \mathbb{R}\right)$-системиl. 
2.3. Обобщенные функции Ляпунова для $(A, h, \mathbb{R})$-системы. Пусть $A$ произвольное абстрактное множество и $S(A)$ - совокупность всех непустых подмножеств множества $A$. Пусть задано отображение $h: A \rightarrow S(A)$ такое, что отношение $y \in h(x)$ является отношением предпорядка, т.е. рефлексивным и транзитивным отношением. Тройка $(A, h, \mathbb{R})$ называется [31] общей системой $c$ заданной структурой следования $h: A \rightarrow S(A)$ (или для краткости $(A, h, \mathbb{R})$-системой). Структуру следования $h$ для краткости будем называть предпорядком. Для $\mathscr{A} \subset S(A)$ определим

$$
h(\mathscr{A}):=\{h(B): B \in \mathscr{A}\}, \quad \text { где } h(B):=\bigcup\{h(\mathscr{A}): \mathscr{A} \in B\} .
$$

Любое непустое подмножество множества $S(A)$ называется квазифильтром на множестве $A$. Пусть $\mathscr{F}$ и $\mathscr{D}$ - квазифильтры на $A$. Будем говорить, что $\mathscr{F}$ сильнее, чем $\mathscr{D}$, или $\mathscr{D}$ слабее, чем $\mathscr{F}$, и писать $\mathscr{D} \prec \mathscr{F}$, если $\forall D \in \mathscr{D} \exists F \in \mathscr{F}$ такое, что $F \subset D$. Квазифильтры $\mathscr{F}$ и $\mathscr{D}$ называются әквивалентными, если $\mathscr{D} \prec \mathscr{F}$ и $\mathscr{F} \prec \mathscr{D}$. Квазифильтр называется допустимымм, если он эквивалентен квазифильтру $\mathscr{B}$ вида $\mathscr{B}=\left\{B_{i}: i \in I\right\}$, где $I$ есть подмножество из $(0, \infty)$, замькание которого содержит нулевую точку, и семейство множеств $\left\{B_{i}\right\}$ неубывающее.

Пусть $h: A \rightarrow S(A)$ - заданное отображение и $\mathscr{F}, \mathscr{D}$ - заданные квазифильтры на некотором множестве $A$. Структура $h$ называется положительно орбитально $(\mathscr{F}, \mathscr{D})$-устойчивой, если $\mathscr{D} \prec H(\mathscr{F})$, и положстельно орбитально $\mathscr{D}$-устойчивой, если она $(\mathscr{D}, \mathscr{D})$-устойчива.

Для заданной функции $V: A \rightarrow[0,+\infty]$ определим множества

$$
\begin{gathered}
B(V, c):=\{A \in x: V(x)<c\}, \quad 0<c \leqslant+\infty, \\
B(V):=\{B(V, c): B(V, c) \neq \varnothing\} .
\end{gathered}
$$

ОПРЕДЕЛЕНИЕ 2.3 [31]. Пусть $\mathscr{F}$ и $\mathscr{D}$ - квазифильтры на $A$. Функция $V: A \rightarrow$ $[0, \infty]$ называется обобщенной функиией Ляпунова относительно $(\mathscr{F}, \mathscr{D})$ для $(A, h, \mathbb{R})$-системы, если выполнены следующие условия:

$$
\begin{gathered}
\mathscr{D} \prec B(V), \\
B(V) \prec \mathscr{F}, \\
\forall c>0 \quad h(B(V, c)) \subset B(V, c) .
\end{gathered}
$$

Условие (2.5) означает, что $V$ является невозрастающей функцией относительно $h$, т.е. $y \in h(x) \Rightarrow V(y) \leqslant V(x)$.

ПреДЛОЖЕНИЕ 2.3 [31]. 1) Если $\mathscr{F}_{1} \prec \mathscr{F}_{2}$ u $\mathscr{D}_{1} \prec \mathscr{D}_{2}$, то положительная орбитальная $\left(\mathscr{F}_{1}, \mathscr{D}_{1}\right)$-устойчивость влечет положсительную орбитальную $\left(\mathscr{F}_{2}, \mathscr{D}_{2}\right)$-устойчивость.

2) Пусть $\mathscr{F}$ и $\mathscr{D}$ - квазифильтры на множестве A. Если существует обобщенная функция Ляпунова относительно $(\mathscr{F}, \mathscr{D})$ для $(A, h, \mathbb{R})$-системь, то структура $h$ является полохсттельно орбитально $(\mathscr{F}, \mathscr{D})$-устойчивой.

3) Если структура $h$ является положстельно орбитально $(\mathscr{F}, \mathscr{D})$-устойчивой и квазифильтр $\mathscr{F}$ (или квазифильтр $\mathscr{D})$ на множсестве $A$ допустим,

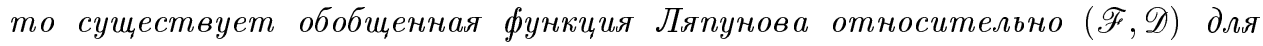
$(A, h, \mathbb{R})$-системы.

Пусть $A$ - топологическое пространство, $h: A \rightarrow S(A)$ - рефлексивное и транзитивное отношение и $\mathscr{F}, \mathscr{D}$-квазифильтры на $A$. Рассмотрим следующие случаи. 
СлучАй 1. Пусть квазифильтр $\mathscr{D}$ является допустимым. Тогда если квазифильтр $\mathscr{F}$ не является допустимым, то имеет место положительная орбитальная устойчивость в смысле Бхатиа [30] замкнутого подмножества $M$. Здесь $\mathscr{F}$ совпадает с полным окрестностным фильтром $\mathscr{N}_{M}$ множества $M$, а $\mathscr{D}$ - с метрическим фильтром $\mathscr{M}_{M}$ множества $M$.

СлучАй 2. Пусть квазифильтр $\mathscr{F}$ является допустимым. Необходимое условие Зубова положительной орбитальной устойчивости компактного множества $M \subset A$ состоит в следуюшем:

$$
\forall x \notin M \quad \exists U \in \mathscr{N}_{M} \quad x \notin h(U) .
$$

В случае $(A, f, \mathbb{R})$-системы свойство (2.6) эквивалентно свойству [14]: множество $M$ не содержит $\alpha$-предельных точек любой орбиты вне $M$.

Если $\mathscr{B}_{M}$ есть квазифильтр $\mathscr{B}_{M}:=\{A \backslash\{x\}: x \notin M\}$, то свойство (2.6) выражает положительную орбитальную $\left(\mathscr{N}_{M}, \mathscr{B} M\right)$-устойчивость. Если $A$-метрическое пространство, то $\mathscr{N}_{M}$ - допустимый квазифильтр, а $\mathscr{B}_{M}$ не является допустимым квазифильтром. Легко проверить, что условия (2.3) и (2.4) определения обобшенной функции Ляпунова принимают следующий вид:

$$
\begin{gathered}
x \notin M \Rightarrow V(x)>0, \\
V\left(x_{n}\right) \rightarrow 0 \text { при } x_{n} \rightarrow M,
\end{gathered}
$$

или

$$
\bar{V}(x):=\varlimsup_{y \rightarrow x} V(y)=0 \quad \forall x \in M .
$$

Из условия (2.5) и условий (2.7) и (2.8) вытекает условие (2.6).

Функция

$$
V(x)=\sup \left\{i \in I: h(x) \cap B_{i}=\varnothing\right\}
$$

в случае метрического пространства $A$ примет вид

$$
V(x)=\rho\left(h^{-1}(x), M\right),
$$

где $h^{-1}$ - обратная к $h$ функция такая, что $x \in h(y) \Leftrightarrow y \in h^{-1}(x)$.

СлучАй 3. Пусть $\mathscr{F}$ и $\mathscr{D}$ - допустимые квазифильтры. Предположим, что $A$ - метрическое пространство, множество $M \subset A$ замкнуто и множество $N \subset M$ компактно. Рассмотрим свойство положительной орбитальной $\left(\mathscr{A}_{M}, \mathscr{B}_{M}\right)$-устойчивости: для любой $\delta$-окрестности $U_{1}$ множества $M$ сушествует окрестность $U_{2}$ множества $N$ такая, что $h\left(U_{2}\right) \subset U_{1}$. В случае компактности $M$ имеем $\mathscr{A}_{M}=\mathscr{B}_{M}$. Легко видеть, что

$$
V(x)=\inf \left\{i \in I: h(x) \subset B_{i}\right\}
$$

и функции

$$
\begin{aligned}
& V_{1}(x)=\sup \{\rho(y, N): y \in h(x)\} \\
& V_{2}(x)=\inf \left\{\rho(y, M): y \in h^{-1}(x)\right\}
\end{aligned}
$$

удовлетворяют условиям (2.3)-(2.5). 
2.4. Обобщенные функции Ляпунова для $(A, \Phi, \mathscr{T})$-системы с запаздыванием. Пусть $A$-пространство сходимости Фреше, т.е. множество, в котором выделен некоторый класс последовательностей (называемых сходящимися), причем каждой последовательности $z_{n}$ этого класса поставлен в соответствие некоторый элемент $z:=\lim _{n \rightarrow \infty} z_{n}$ такой, что

а) если $z_{n}=z \forall n$, то $z_{n} \rightarrow z$,

б) если $z_{n} \rightarrow z$ и $z_{k} \subset z_{n}$, то $z_{k} \rightarrow z$.

Пусть $\Phi$ - множество всех функций $\varphi: \mathscr{T} \rightarrow A$, где $\mathscr{T}:=(\alpha,+\infty)$ и $\alpha<+\infty$ или $\alpha=-\infty$. Возможныл движением на множестве $A$ назьвается любая функция $\varphi: \mathscr{T} \rightarrow A$. Будем говорить, что движение $\varphi(t)$ задано в момент $\tau \in \mathbb{R}^{+}$, если известны все значения $\varphi(t)$ при всех $t \in(\alpha+\tau, \tau]$. Оператор $\varphi^{\prime}:(\alpha, 0] \rightarrow A$ такой, что $\varphi(t)=\varphi^{\prime}(t-\tau), t \in(\alpha+\tau, \tau]$, называется предысторией движения $\varphi(t)$ до момента $\tau \in \mathbb{R}^{+}$. Пусть $\Pi([\alpha, 0], A)$ - множество всех возможных предысторий. Очевидно, что одна и та же предыстория $\varphi^{\prime}:(\alpha, 0] \rightarrow A$ может порождать несколько различных движений в момент $\tau$. Всякое движение $\varphi(t)$, имеюшее в момент $\tau$ предысторию $\varphi^{\prime}(\cdot)$, обозначим через $\varphi\left(\varphi^{\prime}, \tau, t\right)$.

По определению имеем

$$
\varphi\left(\varphi^{\prime}, \tau, t\right):=\varphi^{\prime}(t-\tau), \quad t \in(\alpha+\tau, \tau] .
$$

Если $(\alpha, 0]$ совпадает с точкой 0, то предыстория $\varphi(\cdot)$ называется начальнылм значением движения $\varphi(t)$. Оператор $\varphi^{\prime \prime}:[\tau, \infty) \rightarrow A$ такой, что $\varphi^{\prime \prime}\left(\varphi^{\prime}, \tau, t\right):=$ $\varphi\left(\varphi^{\prime}, \tau, t\right), t \geqslant \tau$, называется историей от момента $\tau \in \mathbb{R}^{+}$движения $\varphi\left(\varphi^{\prime}, \tau, t\right)$. Пусть $U(\tau, A)$ - множество всех историй движения $\varphi \in \Phi$ от момента $\tau \in \mathbb{R}^{+}$.

Тройка $(A, \Phi, \mathscr{T})$ называется [32], [33], [8] общей системой на $A$ с запаздыванием (или для краткости $(A, \Phi, \mathscr{T})$-системой с запаздыванием), где $\Phi$-множество всех отображений $\varphi: \mathbb{R}^{+} \rightarrow A$ таких, что $\varphi_{a}:=\varphi(t+a) \in \Phi, t \in \mathbb{R}^{+}, a \in M \subset \mathbb{R}^{+}$.

Предположим, что существует положительно определенная функция $p: A \times A \rightarrow$ $\mathbb{R}^{+}$, причем

$$
p(\varphi, \psi) \geqslant 0, \quad p(\varphi, \psi)=0 \Leftrightarrow \varphi=\psi \quad \forall \varphi, \psi \in A .
$$

Определим топологию на множестве $\Phi: \mathscr{T} \rightarrow A$

$$
\begin{aligned}
p_{\Phi}(\varphi, \psi):= & \sup \{p(\varphi(t), \psi(t)): t \in \mathscr{T}\} \quad \forall \varphi, \psi \in \Phi(\mathscr{T}, A), \\
& B_{\Phi}(\varphi, \varepsilon):=\left\{\psi \in \Phi: p_{\Phi}(\varphi, \psi)<\varepsilon\right\} .
\end{aligned}
$$

Формулой (2.18) определяется $\varepsilon$-окрестность для любого $\varphi \in \Phi$.

Совокупность всех $\varepsilon$-окрестностей всех элементов $\varphi \in \Phi$ образует базис некоторой топологии на множестве $\Phi$. Аналогично вводятся функции $p_{\Pi}\left(\varphi^{\prime}, \psi^{\prime}\right)$, $p_{U}\left(\varphi^{\prime \prime}, \psi^{\prime \prime}\right)$ и с их помощью топологии на пространствах П: $(\alpha, 0] \rightarrow A, U: \tau \rightarrow A$. Последние пространства будем обозначать через П, $U$.

Движение $\varphi(t) \in \Phi(A, \Phi, \mathscr{T})$-системы с запаздыванием называется [8]

1) положительно устойчивым в смысле Ляпунова, если для любых чисел $\varepsilon>0$ и $\tau \in \mathbb{R}^{+}$сушествует число $\delta(\varepsilon, \tau)$ такое, что

$$
p_{\Pi}\left(\varphi^{\prime}, \psi^{\prime}\right)<\delta \Rightarrow p_{U}\left(\varphi^{\prime \prime}, \psi^{\prime \prime}\right)<\varepsilon,
$$

где $\Pi:=\Pi((\alpha, 0], A), U:=U(\tau, A)$,

2) асимптотически положстельно устойчивым в смысле Ляпунова, если $\varphi(t)$ устойчиво в смысле Ляпунова и сушествует число $\gamma(\tau)>0$ такое, что

$$
p\left(\varphi^{\prime}, \psi^{\prime}\right)<\gamma \Rightarrow \lim _{t \rightarrow \infty} p(\varphi(t), \psi(t))=0 .
$$


ПРЕДЛОЖЕНИЕ 2.4 [8]. $(A, \Phi, \mathscr{T})$-система с запаздъванием может быть задана как совокупность отображсений

$$
F_{\tau}: \Pi \rightarrow U \quad \forall \tau \in \mathbb{R}^{+},
$$

переводящих в момент $\tau \in \mathbb{R}^{+}$предисторию $\varphi^{\prime} \in \Pi$ в историю $\varphi^{\prime \prime} \in U$, при әтом пара $\left(\varphi^{\prime}, \varphi^{\prime \prime}\right), \varphi^{\prime}:(\alpha, 0] \rightarrow A, \varphi^{\prime \prime}:[\tau, \infty) \rightarrow A$, определяет единственное движение $\varphi: \mathscr{T} \rightarrow A(A, \Phi, \mathscr{T})$-системы такое, что

$$
\varphi(t)=\varphi^{\prime}(t-\tau), \text { если } t \leqslant \tau, \quad \text { u } \varphi(t)=\varphi^{\prime \prime}(t), \text { если } t \geqslant \tau .
$$

Рассмотрим положительно определенную на $A \times A$ функцию $V_{\tau, t}: A \times A \rightarrow \mathbb{R}^{+}$, и пусть

$$
V_{\tau}(\varphi, \psi):=\sup \left\{V_{\tau, t}(\varphi(t), \psi(t)): t \geqslant \tau\right\} .
$$

ОПРЕДЕЛЕНИЕ 2.4. Функция $V_{\tau}(\varphi, \psi)$, заданная соотношением (2.23), называется обобщенной функиией Ляпунова для $(A, \Phi, \mathscr{T})$-системь с запаздьванием.

Если в $(A, \Phi, \mathscr{T})$-системе предыстории движений совпадают с их начальными значениями, то $(A, \Phi, \mathscr{T})$-система может быть названа $(A, \Phi, \mathscr{T})$-системой без запаздьввания.

ПРЕДЛОЖЕнИЕ $2.5[8] .(A, \Phi, \mathscr{T})$-система без запаздъцвания может бъть задана как совокупность отображсний

$$
F_{a}: \varphi(a) \rightarrow \varphi_{a}, \quad \varphi \in \Phi, \quad a \in M, \quad \varphi(a) \in H(a),
$$

əдe

$$
H(a):=\{\varphi(a): \varphi \in \Phi, a \in M\} .
$$

Предположим, что для каждого $a \in M$ существует положительно определенная функция $p_{a}$, заданная на $H(a) \times H(a)$, и топология на $H(a)$ порождается функцией $p_{a}$. Функция $p_{a}$ является мерой начальных возмущений, а функция $p-$ мерой последуюших возмушений.

Движение $\varphi \in \Phi(A, \Phi, \mathscr{T})$-системы без запаздывания называется

1) положительно устойчивым в смыслле Ляпунова, если для каждого $a \in \mathscr{T}$ отображение $F_{a}: \varphi(a) \rightarrow \varphi_{a}$ непрерывно по $\varphi$,

2) асимптотически положительно устойчивым в смысле Ляпунова, если $\varphi(t)$ положительно устойчиво в смысле Ляпунова и для всех $\psi(a)$ в некоторой окрестности $\varphi(a)$ имеет место $F_{a}: \psi(t) \rightarrow \varphi_{a}$ при $t \rightarrow+\infty$.

2.5. Обобщенные псевдометрика и метрика для $\Sigma$-системы. Общей системой "вход-выход" (или для краткости $\Sigma$-системой) называется отношение над абстрактными множествами $X$ и $Y: \Sigma \subseteq X \times Y$ [34]. Если $\Sigma: X \rightarrow Y$ является функцией, то $\Sigma$-система называется функциональной. Входящие в определение $\Sigma$-системы множества $X$ и $Y$ характеризуют входные и выходные облекты и называются соответственно входньм и выходньм множествами, а их элементы - входами и выходами. Если входы и выходы являются функциями времени, то $\Sigma$-система называется временно́й. Представление общей системы в виде отношения есть представление в форме "вход-выход". Входы функциональной системы могут рассматриваться как причины, а выходы - как следствия; в этом случае 
входное и выходное множества будем называть множествами причин и следствий (объектами-причинами и объектами-следствиями).

Пусть для заданной $\Sigma$-системы и произвольного множества $\Phi$ функция $R: \Phi \times$ $X \rightarrow Y$ обладает свойством: $(x, y) \in \Sigma \Leftrightarrow \forall c R(c, x)=y$.

Множество $\Phi$ называется множеством (объектов) глобальных состояний $\Sigma$ системы. Элементы множества $\Phi$ называются глобальными состояниями системы. Функция $R$ называется глобальной реакиией $\Sigma$-системы.

Общие динамические системы “вход-выход" и пространства их состояний $\Phi$ определены и изучены в монографиии [34].

Введем понятия обобщенной псевдометрики и обобщенной метрики в динамической $\Sigma$-системе. С этой целью рассмотрим квазифильтр $\mathscr{E} \subset S(\Phi)$ на множестве $\Phi$. Рассмотрим также полную решетку $D$ с наименьшим элементом $O$. Пусть для каждого $\varphi \in \Phi$ определено такое подмножество $D_{\varphi} \subset D$, для которого существует функция $R: \Phi \times M \rightarrow D$ со следуюшими свойствами:

$$
\begin{gathered}
R\left(\varphi, \varphi_{1}\right) \geqslant 0 \text { и } R(\varphi, \varphi)=0, \\
R\left(\varphi, \varphi_{1}\right)=R\left(\varphi_{1}, \varphi\right), \\
R\left(\varphi, \varphi_{2}\right) \leqslant R\left(\varphi, \varphi_{1}\right) \vee R\left(\varphi_{1}, \varphi_{2}\right), \\
\forall \psi \in D_{\varphi} \quad B(\varphi, \psi) \in \mathscr{E}, \quad B(\varphi, \psi):=\left\{\varphi_{1}: R\left(\varphi, \varphi_{1}\right) \leqslant \psi\right\}, \\
\forall \alpha \in \mathscr{E} \varphi \in \alpha \Rightarrow \exists \psi \in D_{\varphi} \varphi \in B(\varphi, \psi) \subset \alpha .
\end{gathered}
$$

Функция $R$, обладающая свойствами (2.26), называется обобщенной псевдометрикой относительно квазифильтра $\mathscr{E}$ и обобщенной метрикой (или расстоянием), если подмножество $D_{\varphi}$ является подрешеткой при каждом $\varphi$.

Имеет место следуюшее предложение [34].

ПРЕДЛОЖЕНИЕ 2.6. 1) Пусть задана динамическая Г-система. Пусть $\Phi-$ произвольное множество и $\mathscr{E}$ - некоторый произвольный, но фиксированныи квазифильтр на множестве $\Phi$. Тогда для $(\Phi, \mathscr{E})$ существует обобщенная псевдометрика $R$ относительно квазифильтра $\mathscr{E}$.

2) Пусть задана динамическая Г-система. Пусть

а) $M \subset \Phi-$ некоторое подмножество множества $\Phi$;

б) $\mathscr{E}$ - некоторый квазифильтр на множсестве $\Phi$;

в) $R: \Phi \times \Phi \rightarrow D-$ обобщенная псевдометрика;

г) $R(M, \varphi)$ определено соотношением

$$
R(M, \varphi):=\inf \left\{R\left(\varphi^{\prime}, \varphi\right), \varphi^{\prime} \in M\right\}
$$

Тогда для подмнохества $M$ существует подмножсество $D_{\varphi_{1}} \subset D$ такое, чmo

$$
\begin{gathered}
R(M, \varphi) \geqslant 0 \quad \text { u } R(M, \varphi)=0, \quad \text { ecлu } \varphi \in M, \\
R\left(M, \varphi_{2}\right) \leqslant R\left(M, \varphi_{1}\right) \vee R\left(\varphi_{1}, \varphi_{2}\right), \\
\forall \psi \psi \in D_{\varphi_{1}} \Rightarrow B(M, \psi) \in N(M), \text { zде } N(M)=\{\alpha: \alpha \in \mathscr{E} \wedge M \subset \alpha\}, \\
\forall \alpha \in N(M) \quad \exists \psi \in D_{\varphi_{1}} \quad M \subset B(M, \psi) \subset \alpha .
\end{gathered}
$$

В [18] с помощью свойств (2.28) введено понятие обобщенной функции Ляпунова и установлен признак устойчивости множества для общих полисистем. 


\section{§3. Орбитальная устойчивость множества и аттракторы в $(A, f, \mathbb{R})$-системе. Обобщенные функции Ляпунова относительно двух окрестностных фильтров}

В этом параграфе вводится понятие орбитальной устойчивости множества в $(A, f, \mathbb{R})$-системе, где $A$ - локально компактное пространство. Это понятие охватывает все известные нам виды устойчивости множества в $(A, f, \mathbb{R})$-системе, а также большинство понятий устойчивости состояния равновесия автономной обыкновенной дифференциальной системы. На базе обобщенных функций Ллпунова установим критерий орбитальной устойчивости относительно совокупности двух фильтров.

3.1. Определения. Пусть $M \subset A$ - замкнутое инвариантное подмножество множества локально компактного метрического пространства $A(A, f, \mathbb{R})$-системы.

Окрестностным фильтром (кратко фильтром) множества $M \subset A(A, f, \mathbb{R})$ системы называется такое семейство $\mathscr{F}$ окрестностей (открытых множеств) множества $M$, что пересечение любых двух элементов семейства принадлежит этому семейству и каждое открытое множество, содержащее элемент семейства, принадлежит семейству. Окрестностный фильтр $\mathscr{F}$ множества $M \subset A$ называется гусmымм, если $\bigcap \bar{F}=M$, где $F \in \mathscr{F}$. Через $\left\{\mathscr{F}^{+}\right\}$будем обозначать множество всех положительно инвариантных фильтров.

Понятия положительного продолжения (или положительной пролонгации) $P_{1}^{+}(x)$ точки $x \in A$ и положительного продолжения $P_{1}^{+}(M)$ множества $M \subset A$ в $(A, f, \mathbb{R})$-системе определяются соответственно равенствами

$$
P_{1}^{+}(x)=\bigcap_{U \in N(x)} H^{+}(U), \quad P_{1}^{+}(M)=\bigcup_{x \in M} P_{1}^{+}(x),
$$

где $N(x)$ - совокупность всех окрестностей точки $x, H^{+}(U)=\overline{C^{+}(U)}$.

Положительное продолжение, или положительная пролонгация, множества $M \subset A \quad(A, f, \mathbb{R})$-системы относительно окрестностного фильтра $\mathscr{F}$ этого множества определяется с помощью формулы

$$
P_{\mathscr{F}}(M)=\bigcap_{N \in \mathscr{F}} \overline{C^{+}(N)}
$$

Имеет место включение

$$
P_{\mathscr{D}}(M) \subset P_{\mathscr{F}}(M)
$$

если $\mathscr{F} \subset \mathscr{D}$, где $\mathscr{F}$ и $\mathscr{D}$ - окрестностные фильтры для $M$.

Для системы двух обыкновенных дифференциальных уравнений понятие пролонгации введено А. Пуанкаре [2]. Для $(A, f, \mathbb{R})$-системы понятие пролонгации изучено в [35], [36].

Фильтр $\mathscr{F}$ множества $M \subset A(A, f, \mathbb{R})$-системы называется

1) топологическим и обозначается через $\mathscr{T}$, если $\mathscr{F}$ - семейство всех открытых множеств, содержащих $M$,

2) метрическим и обозначается через $\mathscr{M}$, если $\mathscr{F}$ - семейство всех открытых шаров $B(M, \varepsilon)$, содержащих $M$.

Множество $M \subset A(A, f, \mathbb{R})$-системы называется 
а) положительно орбитально топологически устойчивым (орбитально $\mathscr{T}$-устойчивым), если для любой окрестности $V$ множества $M$ сушествует окрестность $U$ множества такая, что $C^{+}(U) \subset V$,

б) положительно орбитально метрически устойчивым (орбитально $\mathscr{M}$-устойчивым), если для любого числа $\varepsilon>0$ сушествует число $\delta>0$ такое, что $C^{+}(B(M, \delta)) \subset B(M, \varepsilon)$,

в) положстельно орбитально устойчивым относительно окрестностных

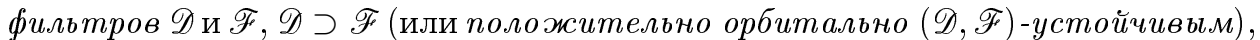
если для каждой окрестности $U \in \mathscr{D}$ множества сушествует окрестность $V \in \mathscr{F}$ множества такая, что $C^{+}(U) \subset V$.

В дальнейшем под устойчивостью всюду будем понимать устойчивость в положительном направлении, опуская для краткости слово “положительный”.

Орбитальная метрическая и орбитальная топологическая устойчивость являются независимыми друг от друга понятиями.

В самом деле, рассмотрим $\left(\mathbb{R}^{2}, f, \mathbb{R}\right)$-систему, определенную уравнениями: $\dot{x}=1, \dot{y}=0$. Очевидно, что $x$-ось орбитально метрически устойчива, но не является орбитально топологически устойчивой. Рассмотрим теперь другую $(A, f, \mathbb{R})$-систему на множестве неотрицательных вещественных чисел, имеющую особые точки во всех точках $x \in \bigcup_{n=0,1,2, \ldots}\left[n, n+2^{-n}\right]$. Пусть $M=\{1,2,3, \ldots\}$. Легко видеть, что множество $M$ орбитально топологически устойчиво, но не является орбитально метрически устойчивым.

Из вышеприведенњых определений следует, что понятия орбитальной топологической и орбитальной метрической устойчивости являются частными случаями понятия орбитальной устойчивости относительно совокупности двух окрестностных фильтров. Орбитальная топологическая (метрическая) устойчивость есть орбитальная устойчивость по совокупности $(\mathscr{F}, \mathscr{F})($ по совокупности $(\mathscr{M}, \mathscr{M}))$.

Определения орбитальной устойчивости индивидуальной полутраектории введены в работах Н. Д. Моисеева [37], Б. П. Демидовича [38], причем Н. Д. Моисеев предложил топологическую версию понятия, а Б.П. Демидович - метрическую версию понятия.

Различные подходы к определению понятия орбитальной устойчивости полуинвариантного и инвариантного (как компактного, так и некомпактного) множества содержатся в работах В.И. Зубова [14], Н.П. Бхатиа и Г. Сегё [30], Ю.В. Малышева [23], Т. Ура [35], Дж. Ауслендера [39], а также в работах [25], [26], [28].

Наиболее общими типами орбитальной устойчивости замкнутого множества $M \subset A(A, f, \mathbb{R})$-системы является орбитальная устойчивость множества относительно совокупности двух метрических окрестностей, относительно совокупности двух топологических окрестностей и относительно совокупности двух окрестностных фильтров множества $M$.

3.2. Критерий орбитальной устойчивости множества относительно двух окрестностных фильтров. Пусть $(\mathscr{D}, \mathscr{F})$ - совокупность двух фильтров $\mathscr{D}$ и $\mathscr{F}$ множества $M, \mathscr{F} \subset \mathscr{D}$, и пусть $N \in \mathscr{D}$ - положительно инвариантное множество $(A, f, \mathbb{R})$-системы. Множество $M$ может быть и некомпактным.

Неотрицательная функция $V: N \rightarrow \mathbb{R}^{+}$назьвается обобщенной функцией Ляпунова $(A, f, \mathbb{R})$-системы для множества $M$ относительно фильтра $\mathscr{D}$, если

a) $V^{-1}(0)=M$

б) $V(f(t, x)) \leqslant V(x) \forall(t, x) \in \mathbb{R}^{+} \times N$, 
в) $V^{-1}([0, c)) \in \mathscr{D}, c>0$.

Семейство $S=S(A)$ неотрицательных функций $V$ из $A$ в $\mathbb{R}^{+}$называется $(\mathscr{D}, \mathscr{F})$-семейством обобщенных функиий Ляпунова $(A, f, \mathbb{R})$-системы для множества $M$, если каждая функция $V$ этого семейства $S$ есть обобщенная функция Ллпунова для множества $M$ относительно $\mathscr{D}$ и для каждой окрестности $F \in \mathscr{F}$ фильтра $\mathscr{F} \subset \mathscr{D}$ сушествует функция $V \in S$ такая, что

$$
\inf \{V(x): x \in U\}>0 \text {. }
$$

Неравенство (3.4) эквивалентно свойству: если $F \in \mathscr{F}$, то существуют функция $V \in S$ и число $d>0$ такие, что $V^{-1}([0, d)) \subset F$.

ЗАмечАниЕ. Обобщенная функция Ляпунова для множества относительно фильтров может и не быть непрерывной.

При совпадении $\mathscr{F}$ и $\mathscr{D}(\mathscr{F}, \mathscr{F})$-семейство обобщенных функций Ляпунова будем называть $\mathscr{F}$-семейством обобшенных функций Ляпунова.

Имеют место следующие теоремы.

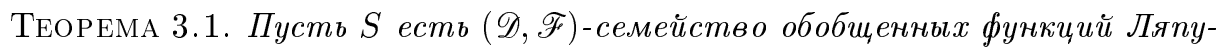
нова $(A, f, \mathbb{R})$-системы для мно жества $M$. Тогда множество $M$ орбитально $(\mathscr{D}, \mathscr{F})$-устойчиво.

ДокАЗАтЕльство. Пусть $F \in \mathscr{F}$ и функция $V \in S$ и число $d>0$ таковы, что $V^{-1}([0, d)) \subset F$. Тогда $D=V^{-1}([0, d)) \in \mathscr{D}$. Если $x \in D, t>0$, то $V(f(t, x)) \leqslant V(x)<d$. Поэтому $f(t, x) \in D$. Следовательно, $C^{+}(D) \subset D \subset F$ и множество $M$ орбитально $(\mathscr{D}, \mathscr{F})$-устойчиво. Теорема 3.1 доказана.

ТЕОРема 3.2. Пусть пространство А удовлетворяет второй аксиоме счетности, и пусть $\mathscr{D}$ и $\mathscr{F}$ - окрестностные фильтры множества $M \subset A$, причем фильтр $\mathscr{F}$ густой. Для того чтобь множсество $M(A, f, \mathbb{R})$-системь

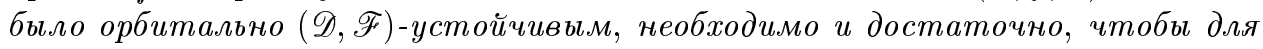
$M$ существовало $(\mathscr{D}, \mathscr{F})$-семейство обобщенных функиий Ляпунова.

ДоКАЗАТЕЛЬство. Пусть множество $M$ является орбитально устойчивым относительно $(\mathscr{D}, \mathscr{F})$. Рассмотрим $F \in \mathscr{F}$ и $x \in F \backslash M$. Выберем окрестность $N_{x} \in \mathscr{D}$ точки $x \in A$ так, чтобы

$$
C^{+}\left(N_{x}\right) \subset F, \quad x \in H^{+}\left(N_{x}\right)
$$

Этот выбор возможен в силу орбитальной устойчивости относительно $(\mathscr{D}, \mathscr{F})$ множества $M$ и в силу свойства густоты фильтра $\mathscr{F}$. Рассмотрим открытую окрестность $U_{x}$ точки $x \in A$ такую, что $U_{x} \subset F, U_{x} \cap H^{+}\left(N_{x}\right)=\varnothing$. Тогда разность $F \backslash M$ есть объединение всех окрестностей $U_{x}$ точки $x \in F \backslash M$. По теореме Линделёфа о счетном покрытии пространства $A$, удовлетворяющего второй аксиоме счетности, существует счетное подпокрытие $\left\{U_{x_{i}}\right\} \subset\left\{\bar{U}_{x}\right\}$ такое, что разность $F \backslash M$ является объединением счетного множества окрестностей $U_{x_{i}}(i=1,2,3, \ldots)$. Определим по индукции множества $A_{i} \in \mathscr{D}: A_{1}=C^{+}\left(N_{x_{1}}\right), \ldots, A_{j}=A_{1} \cap \cdots \cap A_{j-1} \cap$ $H^{+}\left(N_{x_{j}}\right)$. Тогда $A_{1} \supset A_{2} \supset \cdots$ и $\bigcap_{j} \bar{A}_{j}=M$. Определим на $A$ функцию $V(x) \mathrm{c}$ помощью соотношений

$$
V(x)= \begin{cases}\inf \left\{k^{-1}\right\}, & \text { если } C^{+}(x) \subset A_{k}, \\ 1, & \text { если } C^{+}(x) \not \subset A_{k} .\end{cases}
$$


Из определения (3.6) следует, что $V \geqslant 0, V^{-1}(0)=M, V(t, x)=V(x), x \in A$, $t>0$. Каждое множество $A_{k}$ положительно инвариантно: $V(x) \leqslant k^{-1}$, если $x \in A_{k}$. Если $c>0$ и $k^{-1}<c$, то $V^{-1}([0, c)) \supset V^{-1}\left(\left[0, k^{-1}\right)\right) \supset A_{k} \in \mathscr{D}$. Поэтому $V^{-1}([0, c)) \in \mathscr{D}$ и функция $V$ есть обобщенная функция Ляпунова для множества $M$ и фильтра $\mathscr{D}$. Если $x \in F, x \in A_{k}(k=1,2,3, \ldots)$, то $V(x)=1$. Следовательно, для заданной окрестности $F \in \mathscr{F}$ и множества $M$ построена обобшенная функция Ляпунова $V$, которая отграничена от нуля на дополнении множества $F$. Необходимость доказана. Достаточность условий следует из теоремы 3.1. Теорема 3.2 доказана.

Теорема 3.3. Пусть множество $M \subset A(A, f, \mathbb{R})$-системы орбитально $\mathscr{F}$-устойчиво. Если окрестностный фильтр $\mathscr{F}$ множества $M$ имеет счетную базу, то существует भ्F-семейство обобщенных функций Ляпунова, состоящее из одной функиии V. Если фильтр भ्F не имеет счетной базы, то $\mathscr{F}$-семейство обобщенных функиий Ляпунова является несчетным множеством.

ДокАЗАТЕЛЬСтво. Пусть фильтр $\mathscr{F}$ для множества $M$ имеет счетную базу $\left\{F_{1}, F_{2}, \ldots\right\}$, причем $F_{1} \supset F_{2} \supset \cdots$. Предположим, что каждое множество $F_{i}$ положительно инвариантно. Определим обобщенную функцию $V(x):=k^{-1}$, если $x \in F_{k}-F_{k-1}$. Легко видеть, что $\{V\}$ является $\mathscr{F}$-семейством обобшенных функций Ляпунова. Если $\left\{V_{1}, V_{2}, \ldots\right\}$ является счетньп $\mathscr{F}$-семейством функций, то счетной базой фильтра $\mathscr{F}$ будет семейство $\left\{V_{i}^{-1}([0,1 / k)): i, k=1,2, \ldots\right\}$. Итак, сушествует $\mathscr{F}$-семейство $\{V\}$, состояшее из одной функции в случае наличия у фильтра $\mathscr{F}$ счетной базы. Очевидно, что $\mathscr{F}$-семейство $\{V\}$ состоит из несчетного числа функций в случае, когда фильтр $\mathscr{F}$ не обладает счетной базой. Теорема 3.3 доказана.

Отметим, что для некомпактного множества $M \subset A \quad(A, f, \mathbb{R})$-системы существования одной обобшенной функции Ляпунова недостаточно для его устойчивости относительно $\mathscr{F}$, так как в общем случае фильтр $\mathscr{F}$ не имеет счетной базы.

3.3. Аттракторы и обобщенные функции Ляпунова относительно двух окрестностных фильтров. Пусть $\mathscr{D}$ и $\mathscr{F}$ - окрестностные фильтры множества $M(A, f, \mathbb{R})$-системы. Множество $M$ называется положительным аттрактором относительно $\mathscr{D}$ и $\mathscr{F}$ (или положительнымм $(\mathscr{D}, \mathscr{F})$-аттрактором), если сушествует $D_{0} \in \mathscr{D}^{+}$такое, что если $x \in D_{0}, F \in \mathscr{F}$, то сушествует число $\tau=\tau(x, F)$ такое, что $f(t, x) \in F \forall t \geqslant \tau$.

Наибольшее из множеств $M$ называется областью притяжения при $t \rightarrow+\infty$ относительно фильтров $\mathscr{D}$ и $\mathscr{F}$ (или областью $(\mathscr{D}, \mathscr{F})$-притяжения при $t \rightarrow+\infty)$. Если число $\tau$ зависит только от $F$, то множество $M$ назьвается равномерныц положительныц $(\mathscr{D}, \mathscr{F})$-аттрактором.

В дальнейшем под аттракторами будем понимать аттракторы при $t \rightarrow+\infty$, опуская для краткости слово "положительньй".

Множество $M$ называется асимптотически орбитально устойчивым относительно фильтров $\mathscr{D}$ и $\mathscr{F}$ (или асимптотически орбитально $(\mathscr{D}, \mathscr{F})$-устойчивыцм $)$, если оно является $(\mathscr{D}, \mathscr{F})$-аттрактором и орбитально $(\mathscr{D}, \mathscr{F})$-устойчивым.

Из определений $(\mathscr{D}, \mathscr{F})$-аттрактора, $\omega$-предельного множества $\omega(x)$ и из свойства густоты фильтра $\mathscr{F}$ вытекают следующие предложения. 
ПрЕДЛОЖЕНИЕ 3.1. Пусть множество $M \subset A(A, f, \mathbb{R})$-системы есть $(\mathscr{D}, \mathscr{F})$-аттрактор, а фильтр $\mathscr{F}$ густой. Если $x \in D$, то $\omega$-предельное множество $\omega(x)$ является подмножеством множества $M$ (возможно, пус$m b l \cdot M)$.

ПрЕДЛОЖЕНИЕ 3.2. Пусть множсество $M \quad(A, f, \mathbb{R})$-системы есть равномерный $(\mathscr{D}, \mathscr{F})$-аттрактор. Если выполнено $f(t, D) \in \mathscr{D} \forall t \geqslant 0$, то множество $M$ является асимптотически орбитально $(\mathscr{D}, \mathscr{F})$-устойчивым.

ПримеР. Пусть задано нестационарное $n$-мерное векторное обыкновенное дифференциальное уравнение

$$
\frac{d x}{d t}=F(t, x), \quad F(t, 0)=0, \quad t \in \mathbb{R},
$$

такое, что для любого $\left(t_{0}, x_{0}\right) \in \mathbb{R} \times \mathbb{R}^{n}$ сушествует единственное решение $f\left(t, t_{0}, x_{0}\right), f\left(t_{0}, t_{0}, x_{0}\right)=0$, определенное для всех значений $t \in \mathbb{R}$. Сопоставим уравнению (3.7) стационарное $(n+1)$-мерное векторное уравнение

$$
\frac{d y}{d t}=G(y), \quad y=(t, x), \quad G=(1, F)
$$

Решения уравнения (3.7), определенные в $\mathbb{R}^{n+1}$, образуют $\left(\mathbb{R}^{n+1}, f, \mathbb{R}\right)$-систему, в которой $M=\{(t, 0): t \in \mathbb{R}\}$ есть замкнутое инвариантное множество. Под окрестностью множества $M$ будем понимать множество $\{(t, x): t \in \mathbb{R},|x|<\delta(t, \varepsilon)\}$. Легко проверить, что решение $x=0$ уравнения (3.7)

1) устойчиво в смысле Ляпунова тогда и только тогда, когда множество $M$ является орбитально $(\mathscr{T}, \mathscr{M})$-устойчивым,

2) равномерно устойчиво в смысле Ляпунова тогда и только тогда, когда множество $M$ является орбитально $\mathscr{M}$-устойчивым.

Кроме того, легко проверить, что решение $x=0$ уравнения (3.7)

3) квазиасимптотически устойчиво (асимптотически устойчиво) в смысле Ляпунова тогда и только тогда, когда множество $M$ является $(\mathscr{T}, \mathscr{M})$-аттрактором (орбитально асимптотически $(\mathscr{T}, \mathscr{M})$-устойчивьм),

4) равномерно квазиасимптотически устойчиво (равномерно асимптотически устойчиво), если множество $M$ является $\mathscr{M}$-аттрактором (асимптотически $\mathscr{M}$-устойчивым).

Определения вьшеприведенных типов устойчивости решения $x=0$ уравнения (3.7) даны в [40].

Если $x_{n}$ - последовательность в $A$ и $\mathscr{D}$ - окрестностный фильтр множества $M$, то запись $x_{n} \stackrel{\mathscr{D}}{\longrightarrow} M$ ( “ $x_{n}$ по фильтру $\mathscr{D}$ стремится к $M$ ") будет означать, что $x_{n}$ в конце концов окажется в каждом множестве $D \in \mathscr{D}$.

ЛЕмма 3.1. Пусть $x_{n} \stackrel{\mathscr{D}}{\longrightarrow} M u V$ есть $\mathscr{D}$-семейство обобщенных функиий Ляпунова $(A, f, \mathbb{R})$-системы для $M$. Тогда $V\left(x_{n}\right) \rightarrow 0$.

ДокАЗАтЕльство. В самом деле, если $S-(\mathscr{D}, \mathscr{F})$-семейство обобщенных функций Ляпунова для $M$, то из $x_{n} \stackrel{\mathscr{D}}{\longrightarrow} M$ вытекает, что $V\left(x_{n}\right) \rightarrow 0$ и, следовательно, $x_{n} \stackrel{\mathscr{F}}{\longrightarrow} M$. Поэтому $S$ есть $\mathscr{F}$-семейство обобшенных функций Ляпунова для $M$. Следовательно, $x_{n} \stackrel{\mathscr{F}}{\longrightarrow} M$ тогда и только тогда, когда $V\left(x_{n}\right) \rightarrow 0$ для всех $V$ из семейства $S$. Лемма 3.1 доказана.

Имеет место следующая 
TEOPEMA 3.4. Пусть

1) множество $M \subset A \quad(A, f, \mathbb{R})$-системь орбитально $\mathscr{F}$-устойчиво и

2) $S$ есть $\mathscr{F}$-семейство обобщенных функций Ляпунова для $M$.

Для того чтобы множество $M$ было орбитально асимптотически $\mathscr{F}-у с-$ тойчиво, необходимо и достаточно, чтобъ

$$
\exists F \in \mathscr{F}^{+} \quad \lim _{t \rightarrow+\infty} V(f(t, x))=0 \quad \forall x \in F .
$$

ДокАЗАТЕльство. Утверждение теоремы есть следствие эквивалентности двух соотношений $x_{n} \stackrel{\mathscr{F}}{\longrightarrow} M$ и $V\left(x_{n}\right) \rightarrow 0, \quad V \in S$, для последовательностей $x_{n}$ в $A$ (см. лемму 3.1 ). Теорема 3.4 доказана.

Топологический аттрактор характеризуется следующей теоремой.

TЕОРемА 3.5. Пусть $M$ - замкнутое инвариантное множество $(A, f, \mathbb{R})$ системы. Тогда следующие свойства әквивалентны:

$\left(P_{1}\right) M-$ - топологический аттрактор;

$\left(P_{2}\right)$ существует окрестность $U$ множества $M$ такая, что замыкание $\overline{C^{+}(x)}$ компактно для каждой точки $x \in U \backslash M u \omega(x) \subset M$;

$\left(P_{3}\right) M-\mathscr{F}$-аттрактор для некоторого густого окрестностного фильтра $\mathscr{F}$ и замыкание $\overline{C^{+}(x)}$ компактно для всех точек $x$ в некоторой окрестности $U$ мнолества $M$.

ДокаЗАТЕЛЬСтво. Очевидно, что

$$
\left(P_{2}\right) \Rightarrow\left(P_{1}\right) \text { и }\left(P_{3}\right) \Rightarrow\left(P_{2}\right) .
$$

Покажем, что $\left(P_{1}\right) \Rightarrow\left(P_{2}\right)$. В самом деле, если $\left(P_{2}\right)$ не имеет места, то сушествует последовательность $x_{i}$ такая, что $x_{i} \in M, \quad x_{i} \rightarrow x \in M$ и для которой либо

$1)$ траектория $C^{+}\left(x_{i}\right)$ некомпактна, либо

2) сушествует $y_{i} \in \omega\left(x_{i}\right) \backslash M$.

В обоих случаях можно предположить, что $x_{i} \in U$. В первом случае для каждого $i$ сушествует последовательность $t_{n_{i}}$ такая, что последовательность $f\left(t_{n_{i}}, x_{i}\right)$ $(n=1,2, \ldots)$ не имеет сходящейся подпоследовательности. Пусть $U_{i}=A \backslash$ $\bigcup_{j} f\left(t_{n_{j}}, x_{i}\right)$. Тогда каждое множество $U_{i}$ есть окрестность $M$ и свойство притяжения отсутствует. Во втором случае поступаем аналогично. А именно выберем последовательность $t_{n_{i}}$ так, чтобы $t_{n_{i}} \rightarrow \infty$ при $n_{i} \rightarrow \infty$ и $f\left(t_{n_{i}}, x_{i}\right) \rightarrow y_{i} \in M$. Обозначая, как и прежде, $U_{i}=A \backslash \bigcup_{j} f\left(t_{n_{j}}, x_{i}\right)$, приходим к отсутствию свойства притяжения. Наконец, очевидны импликации $\left(P_{1}\right) \Rightarrow\left(P_{3}\right)$ и $\left(P_{2}\right) \Rightarrow\left(P_{3}\right)$. Tеорема 3.5 доказана.

3.4. Связь между топологическим и метрическим аттракторами. Пусть $\mathscr{F}$ - окрестностный фильтр множества $M \subset A$. Если сушествует окрестность $U \in \mathscr{F}$ такая, что для любого $V \in \mathscr{F}$ и любой точки $p \in U$ соотношение $f([\tau, \infty), p) \subset U$ справедливо для достаточно больших $\tau=\tau(p, V)$, то множество $M$ называется топологическим аттрактором. Максимальная окрестность $U$ с указанньм свойством называется областью топологического притяжения $M$ и обозначается через $\Pi^{t}(M)$.

Множество $\Pi^{m}(M)$ метрического притяжения $M$ есть множество всех точек $x$ таких, что $\rho(f(t, x), M) \rightarrow 0$ при $t \rightarrow+\infty$. Если $\Pi^{m}(M) \in \mathscr{F}$, где $\mathscr{F}-$ 
фильтр множества $M$, то множество $M$ называется метрическим аттрактором $(A, f, \mathbb{R})$-системы.

Имеет место следуюшая очевидная

ТЕОРема 3.6. Пусть $L^{+}$есть обвединение всех устойчивых в смысле Лагранжа полутраекторий $(A, f, \mathbb{R})$-системы. Междумножествами $\Pi^{t}(M)$ и $\Pi^{m}(M)$ топологического и метрического притяэсения замкнутого множества $M \subset A \quad(A, f, \mathbb{R})$-системы и множеством $L^{+}$имеется следующая связи:

$$
\Pi^{t}(M)=\Pi^{m}(M) \cap L^{+}
$$

СЛЕДСТВИЕ 3.1. Замкнутое множество $M \subset A \quad(A, f, \mathbb{R})$-системы есть топологический аттрактор тогда и только тогда, когда $M$ является метрическим аттрактором и $L^{+} \in \mathscr{F}$, әде $\mathscr{F}$ - окрестностный фильтр множсесmва $M \subset A$.

\section{$\S 4$. Орбитальная устойчивость в $(A, h, \mathbb{R})$-системе и обобщенные функции Ляпунова относительно двух базисов фильтров}

В этом параграфе изучено понятие орбитальной устойчивости относительно двух базисов фильтров для $(A, h, \mathbb{R})$-системы, заданной структурой следования $h$. Это определение охватьвает понятие устойчивости при постоянно действующих возмущениях, понятие устойчивости одного множества относительно другого, понятие устойчивости по двум мерам. Установлен критерий орбитальной устойчивости на базе обобшенных функций Ляпунова относительно двух базисов фильтров.

4.1. Орбитальная устойчивость по двум базисам фильтров. Рассмотрим $(A, h, \mathbb{R})$-систему на множестве $A$ (определения см. в $\S 2$ ). Пусть $A$ - абстрактное множество, $S(A)$ - множество всех непустых подмножеств множества $A$ и $S(f)$ - множество всех отображений $f: A \rightarrow S(A)$.

Обозначим через $\mathscr{E}$ (соответственно через $\mathscr{F}$ ) квазифильтр относительно множества $A$ (множества $S(f))$. Если пересечение любых двух элементов множества $\mathscr{E}($ множества $\mathscr{F})$ принадлежит множеству $\mathscr{E}$ (множеству $\mathscr{F}$ ), то множество $\mathscr{E}$ (множество $\mathscr{F}$ ) называется базисом фильтра на $A($ на $S(f))$.

Пусть $\mathscr{F}=\{F\}$ и $\mathscr{E}=\{E\}$ суть базисы фильтров соответственно на $S(f)$ и $A$. Базис фильтра $\mathscr{F}(\mathscr{E})$ на множестве $A$ определяется следующим образом:

$$
\mathscr{F}(\mathscr{E})=\left\{F(E): F(E)=\bigcup_{h \in F, x \in E} h(x)\right\} .
$$

Пусть $\mathscr{E}_{1}$ и $\mathscr{E}_{2}-$ базисы фильтров на $A$. Будем говорить, что базис $\mathscr{E}_{1}$ слабее базиса $\mathscr{E}_{2}$ и писать $\mathscr{E}_{1} \prec \mathscr{E}_{2}$, если для любого $E_{1} \in \mathscr{E}_{1}$ существует $E_{2} \in \mathscr{E}_{2}$ такое, что $E_{2} \subseteq E_{1}$. Если же имеет место как $\mathscr{E}_{1} \prec \mathscr{E}_{2}$, так и $\mathscr{E}_{2} \prec \mathscr{E}_{1}$, то базисы фильтров $\mathscr{E}_{1}$ и $\mathscr{E}_{2}$ будем называть әквивалентнылми.

Очевидно, что если $\mathscr{E}_{1} \prec \mathscr{E}_{2}$ и $\mathscr{E}_{2} \prec \mathscr{E}_{3}$, то $\mathscr{E}_{1} \prec \mathscr{E}_{3}$, т.е. отношение $\prec$ является транзитивным. 
ОПреДЕЛЕНИЕ 4.1. Пусть $\mathscr{E}_{1}$ и $\mathscr{E}_{2}-$ базисы фильтров на множестве $A$, а $\mathscr{F}-$ базис фильтра на $S(f)$. Пара $(A, \mathscr{F})(A, h, \mathbb{R})$-системы называется орбитально $\left(\mathscr{E}_{1}, \mathscr{E}_{2}\right)$-устойчивой, если $\mathscr{E}_{1} \prec \mathscr{F}\left(\mathscr{E}_{2}\right)$.

ОПРЕДЕЛЕНИЕ 4.2. Пусть $Г$ - некоторое частично упорядоченное множество с отношением предпорядка $\leqslant, \mathscr{F}$ - базис фильтра на $S(f)$ и $\mathscr{E}_{1}, \mathscr{E}_{2}-$ базисы фильтров на множестве $A$. Функция $V: A \rightarrow \Gamma$ называется обобщенной функиией Ляпунова для $(A, h, \mathbb{R})$-системы, если сушествуют подмножества $\Gamma_{1}$ и $\Gamma_{2}$ множества $\Gamma$ такие, что множества

$$
B\left(\Gamma_{i}\right)=\left\{B(c, V), c \in \Gamma_{i}\right\}, \quad B(V)=\{x \in A: V(x) \leqslant c\}
$$

яВляются базисами фильтров, причем

$$
\begin{gathered}
\mathscr{E}_{1} \prec B\left(\Gamma_{1}\right), \quad B\left(\Gamma_{2}\right) \prec \mathscr{E}_{2}, \\
B\left(\Gamma_{1}\right) \prec \mathscr{F}\left(B\left(\Gamma_{2}\right)\right) .
\end{gathered}
$$

Определение 4.2 является обобщением определения 2.3 из § 2 .

Имеет место следующая

Teорема 4.1. Пусть $\mathscr{F}$ - базис фильтра на $S(f), \mathscr{E}_{1}, \mathscr{E}_{2}-$ базисы фильтров на $A$. Для того чтобъ пара $(A, \mathscr{F})(A, h, \mathbb{R})$-системы была орбитально $\left(\mathscr{E}_{1}, \mathscr{E}_{2}\right)$-устойчивой, необходимо и достаточно, чтобъ для этой системь существовала обобщенная функиия Ляпунова, обладающая свойствами (4.3) $u(4.4)$.

ДоКАЗАТЕЛЬСТво. Множество $S(A)$ упорядочим по вкЛючению и примем его в качестве Г. Пусть

$$
V(x):= \begin{cases}\bigcap\left\{e: x \in e, e \in \mathscr{E}_{1} \cup \mathscr{E}_{2}\right\}, & \text { если существует } \\ & e \in \mathscr{E}_{1} \cup \mathscr{E}_{2} \text { такое, что } x \in e ; \\ \sup _{A} S(A) & \text { в противном случае. }\end{cases}
$$

Рассмотрим совокупность подмножеств

$$
B(e, V)=\{x \in A: V(x) \leqslant e\}, \quad e \in \mathscr{E}_{1} \cup \mathscr{E}_{2} .
$$

В силу определений $V(x)$ и $B(e, V)$ имеет место

$$
B(e, V)=e \quad \forall e \in \mathscr{E}_{1} \cup \mathscr{E}_{2}
$$

Положим $\Gamma_{1}=\mathscr{E}_{1}, \Gamma_{2}=\mathscr{E}_{2}$. Тогда $B\left(\Gamma_{1}\right)$ являются базисами фильтров со свойством (4.3). Так как пара $(A, \mathscr{F})$ является орбитально $\left(\mathscr{E}_{1}, \mathscr{E}_{2}\right)$-устойчивой, то из (4.5) следует свойство (4.4). Необходимость доказана. Достаточность вытекает из свойств (4.3) и (4.4) и свойства транзитивности отношения $\leqslant$. Теорема 4.1 доказана. 
4.2. Устойчивость при постоянно действующих возмущениях. Пусть на множестве $A \subset \mathbb{R}^{n}$ евклидова пространства $\mathbb{R}^{n}$ задано стационарное дифференциальное уравнение $\dot{x}=f(x)$. Будем предполагать, что вьполнены условия теоремы о сушествовании и единственности решения уравнения и $f(0)=0$.

Решение $x=0$ называется устойчивым при постоянно действующих возмущения $x$, если для произвольного $\varepsilon>0$ найдутся $\delta(\varepsilon)>0$ и $\eta(\varepsilon)>0$ такие, что для любой непрерывно дифференцируемой функции $\widetilde{x}(t)$ выполняется $\|\widetilde{x}(t)\|<\varepsilon$ при $t \geqslant 0$, лишш только $\|\widetilde{x}(0)\|<\delta(\varepsilon)$ и $\|\dot{\widetilde{x}}(t)-f(\widetilde{x}(t))\|<\eta(\varepsilon)$.

В данном случае $A \subset \mathbb{R}^{n}$, и если базис фильтра $\mathscr{F}=\left\{F_{\eta}\right\}$ на $S(f)$ состоит из отображений вида

$$
F_{\eta}=\left\{\widetilde{x}(0) \rightarrow C_{\widetilde{x}(0)}:\|\dot{\widetilde{x}}(t)-f(\widetilde{x}(t))\|<\eta\right\}, \quad 0<\eta<\infty,
$$

где $C_{\widetilde{x}}(0)$ - положительная полутраектория движения $\widetilde{x}(t)$, проходящего при $t=0$ через $\widetilde{x}(0)$, а $\mathscr{E}_{1}$ и $\mathscr{E}_{2}$ являются базисами фильтров окрестностей точки $x=0$ в $A \subset \mathbb{R}^{n}$, то определение 4.1 приводит к определению устойчивости при постоянно действующих возмущениях.

4.3. Орбитальная устойчивость множества относительно другого множества. Инвариантное множество $Y \subseteq A$ непрерывной группы преобразований $(A, T, h)$ назьвается орбитально устойчивым относительно множества $Z \subseteq A$, если для произвольной окрестности $U$ множества $Y$ существует окрестность $W$ множества $Y$ такая, что образы точек из $W \cap Z$ при всех преобразованиях групшы $h$ лежат в $U$.

Здесь в качестве базиса фильтра $\mathscr{1} 1$ можно взять базис фильтров окрестностей множества $Y$, а в качестве $\mathscr{E}_{2}$ - базис фильтра, индуцированный в $Z$ базисом $\mathscr{E} 1$ и $\mathscr{F}=\{h\}$.

4.4. Устойчивость по двум метрикам. Пусть решение задается функцией $x(p, t), p \in A \subset \mathbb{R}^{n}, t \in[0,+\infty]$, удовлетворяюшей соотношению $\frac{d x}{d t}=G[x]$ и заданным начальным и граничным условиям, где $G$ - некоторый оператор, причем $G[0]=0$. Рассмотрим вешественную функцию $\rho[x(p, t)]$, определенную для каждого фиксированного момента времени $t \geqslant 0$ на множестве функций $A=\{x(p)\}$, удовлетворяющую условиям:

a) $\rho[x(p, t)] \geqslant 0$

б) $\rho[0]=0$;

в) для любого $x(p, t)$ вешественная функция $\rho[x(p, t)]$ от аргумента $t$ непрерьвна по $t$.

Решение $x(p, t)$ назьвается равномерно устойчивьм по метрикам $\rho_{1} u \rho_{2}$, если для произвольного $\varepsilon>0$ существует $\delta(\varepsilon)>0$ такое, что из $\rho_{2}[x(p, 0)]<\delta(\varepsilon)$ следует $\rho_{1}[x(p, t)]<\varepsilon$ при $t \geqslant 0$.

Взяв в качестве базисов фильтров

$$
\begin{array}{lll}
\mathscr{E}_{1}=\left\{E_{1, \varepsilon}\right\}, & \text { где } E_{1, \varepsilon}=\left\{x(p): \rho_{1}[x(p)]<\varepsilon\right\}, & 0<\varepsilon<\infty, \\
\mathscr{E}_{2}=\left\{E_{2, \varepsilon}\right\}, & \text { где } E_{2, \varepsilon}=\left\{x(p): \rho_{2}[x(p)]<\delta\right\}, & 0<\delta<\infty,
\end{array}
$$


а в качестве $\mathscr{F}=\{F\}$ отображение

$$
F=\left\{x(p, 0) \rightarrow C_{x(p, 0)}: \frac{\partial x(p, t)}{\partial t}=G[x(p, t)]\right\},
$$

удовлетворяющее краевым условиям, где $C_{x(p, 0)}-$ множество функций $x(p)$ вида $x(p, t)$ при каждом фиксированном $0<t<\infty$, получим определение устойчивости по двум метрикам.

$\S 5$. Орбитальная устойчивость в $(A, h, \mathbb{R})$-системе и обобщенные функции Ляпунова относительно квазифильтров

В этом параграфе введено понятие орбитальной устойчивости относительно двух квазифильтров в $(A, h, \mathbb{R})$-системе с заданной структурой предпорядка $h$ и установлен критерий орбитальной устойчивости на базе обобщенных функций Ляпунова относительно квазифильтров.

5.1. Критерий орбитальной устойчивости относительно квазифильтров. Рассмотрим $(A, h, \mathbb{R})$-систему с заданной структурой предпорядка. Пусть $\mathscr{F}$ и $\mathscr{D}$ - квазифильтры на множестве $A$ со структурой $(A, h)$. Понятие орбитальной устойчивости структуры предпорядка дано в $\S 2$. Переформулируем определение этого понятия следующим образом.

Пусть $h: A \rightarrow S(A)$ - заданное отображение, $\mathscr{F}$ и $\mathscr{D}$ - заданные квазифильтры на множестве $A$. Структура $(A, h)$ называется

1) орбитально $(\mathscr{F}, \mathscr{D})$-устойчивой, если для любого заданного множества $D \in \mathscr{D}$ существует множество $F \in \mathscr{F}$ такое, что $h(F) \subset D$,

2) орбитально $\mathscr{F}$-устойчивой, если она орбитально $(\mathscr{F}, \mathscr{F})$-устойчива.

Понятие обобщенной функции Ляпунова дано в $\S 2$. Напомним, что функция $V: A \rightarrow[0, \infty)$ называется обобщенной функцией Ляпунова относительно квазифильтров $\mathscr{F}$ и $\mathscr{D}$ для $(A, h, \mathbb{R})$-системы, если выполнены условия (2.3)-(2.5).

Имеет место следующая

Лемма 5.1. Пусть $\mathscr{F}, \mathscr{G}, \mathscr{D}$ - квазифильтры $(A, h, \mathbb{R})$-системы на множестве $A$. Орбитальная $\mathscr{G}$-устойчивость структурь предпорядка $(A, h)$ вле-

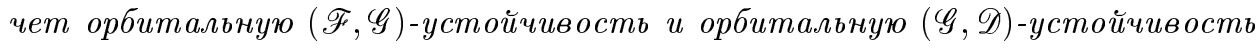

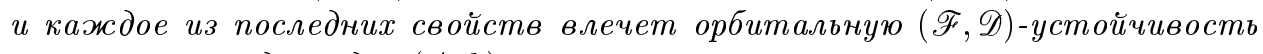
структуры предпорядка $(A, h)$, если выполнены условия

$$
\mathscr{D} \prec \mathscr{G} \prec \mathscr{F} \text {. }
$$

Доказательство леммы 5.1 опирается на соотношения (5.1) и тот факт, что если $\mathscr{D}_{1} \prec \mathscr{D}_{2}$ и $\mathscr{F}_{1} \prec \mathscr{F}_{2}$, то из орбитальной $\left(\mathscr{F}_{1}, \mathscr{D}_{1}\right)$-устойчивости вытекает орбитальная $\left(\mathscr{F}_{2}, \mathscr{D}_{2}\right)$-устойчивость.

Имеет место следуюшая

ТеОрема 5.1. Пусть $\mathscr{F}$ и $\mathscr{D}$ - квазифильтры множсества $A$ и $\mathscr{G}$-допустимый квазифильтр такой, что имеет место (5.1). Для того чтобъ структура предпорядка $(A, h)$ для $(A, h, \mathbb{R})$-системы была одной из трех типов:

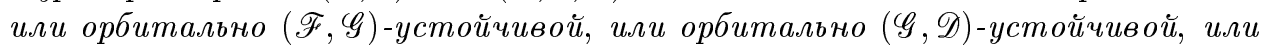
орбитально $\mathscr{G}$-устойчивой, необходимо и достаточно, чтобь существовала обобщенная функция Ляпунова $V: A \rightarrow \mathbb{R}^{+}$этой системы относительно квазифильтров $\mathscr{F}$ и $\mathscr{D}$. 
ДокАЗАТЕЛЬСтво. Достаточность. Предположим, что $V$ есть обобщенная функция Ляпунова относительно $(\mathscr{F}, \mathscr{D})$ для $(A, h, \mathbb{R})$-системы. Положим $\mathscr{G}=B(V)$. Тогда (5.1) есть следствие условий

$$
\mathscr{D} \prec B(V), \quad B(V) \prec \mathscr{F} .
$$

Из $h(B(V, c)) \subset B(V, c)$ следует, что $B(V) \prec h(B(V))$. Следовательно, из условия орбитальной $\mathscr{G}$-устойчивости вытекают орбитальная $(\mathscr{F}, \mathscr{G})$-устойчивость или орбитальная $(\mathscr{G}, \mathscr{D})$-устойчивость.

Необходимость. Предположим, что квазифильтр $\mathscr{G}$ является допустимым и выполнено условие (5.1). Если структура $(A, h)$ является орбитально $(\mathscr{F}, \mathscr{G})$-устойчивой, то в силу предложения 2.3 сушествует обобщенная функция Ляпунова $V$ для $(A, h, \mathbb{R})$ и, следовательно, будут выполнены условия

$$
\mathscr{G} \prec B(V) \prec \mathscr{F} \text { и } h(B(V, c)) \subset B(V, c), \quad c>0 .
$$

Из условий (5.1) и (5.3) следуют условия

$$
\begin{gathered}
\mathscr{D} \prec B(V), \quad B(V) \prec \mathscr{F}, \\
\forall c>0 \quad h(B(V, c)) \subset B(V, c) .
\end{gathered}
$$

Поэтому $V$ есть обобщенная функция Ляпунова относительно $(\mathscr{F}, \mathscr{D})$ для $(A, h, \mathbb{R})$. Если же структура предпорядка $(A, h)$ является $(\mathscr{G}, \mathscr{D})$-устойчивой, то из предложения 2.3 следует существование обобшенной функции Ляпунова $V$ относительно $(\mathscr{G}, \mathscr{D})$ для $(A, h, \mathbb{R})$-системы и, таким образом, выполнены условия

$$
\mathscr{D} \prec B(V) \prec \mathscr{G} \text { и } h(B(V, c)) \subset B(V, c) .
$$

Из условий (5.1) и (5.6) следуют условия $(5.4),(5.5)$. Случай $\mathscr{G}$-устойчивости рассматривать нет необходимости, так как $\mathscr{G}$-устойчивость влечет как $(\mathscr{F}, \mathscr{G})$-устойчивость, так и $(\mathscr{G}, \mathscr{D})$-устойчивость, и каждая из них влечет $(\mathscr{F}, \mathscr{D})$-устойчивость. Теорема доказана.

5.2. Орбитальная $\left(\mathscr{E}_{1}, \mathscr{E}_{2}\right)$-устойчивость. Рассмотрим $(A, f, \mathbb{R})$-систему на метрическом пространстве $A$ и замкнутое непустое множество $M \subset A$. Окрестностный фильтр множества $M$ обозначим через $\mathscr{E}_{1}$ и рассмотрим квазифильтр

$$
\mathscr{E}_{2}:=\{A \backslash\{x\}: x \in A \backslash M\},
$$

яВляюшийся квазифильтром окрестностного фильтра $\mathscr{E}_{1}$.

Замкнутое множество $M$ является орбитально $\left(\mathscr{E}_{1}, \mathscr{E}_{2}\right)$-устойчивым, если для любой точки $x \notin M$ существует окрестность $U$ множества такая, что

$$
x \notin C^{+}(U) .
$$

Легко показать, что для орбитальной $(\mathscr{E} 1, \mathscr{E} 2)$-устойчивости множества $M$ достаточно сушествования непрерывной на $M$ функции $V$, обладаюшей свойствами

$$
V>0 \quad \forall x \in A \backslash M
$$


и

$$
V=0 \quad \forall x \in M
$$

Предположим, что орбитально $\left(\mathscr{E}_{1}, \mathscr{E}_{2}\right)$-устойчивое множество содержит компактный глобальный аттрактор $Q$ относительно $M$, т.е. такое компактное множество $Q$, что все положительные полутраектории в $M$ стремятся при $t \rightarrow+\infty$ к $Q$. Кроме того, предположим, что $Q$ не содержит положительных предельных точек траекторий вне $M$. Квазифильтр $\mathscr{G}:=\left\{G_{c}: c>0\right\}$, где $G_{c}=\{x \in A$ : $\rho(C(x), Q)<c\}$, является допустимым и удовлетворяет при $\mathscr{F}=\mathscr{E}_{1}$ и $\mathscr{D}=\mathscr{E}_{2}$ условию $(5.1)$ и условию орбитальной $(\mathscr{G}, \mathscr{G})$-устойчивости. Следовательно, существует обобщенная функция Ляпунова

$$
V(x):=\inf \left\{c: x \in G_{c}\right\}=\rho(C(x), Q),
$$

удовлетворяющая условиям (5.9) и (5.10).

\section{§ 6. Устойчивость в смысле Ляпунова в $(A, \Phi, \mathscr{T})$-системе с запаздыванием и обобщенные функции Ляпунова относительно двух метрик}

В этом параграфе установлены критерии устойчивости и асимптотической устойчивости в $(A, \Phi, \mathscr{T})$-системе с запаздьванием на базе обобщенных функций Ляпунова относительно двух метрик.

Имеют место следующие критерии устойчивости и асимптотической устойчивости движения $\varphi(t) \in \Phi(A, \Phi, \mathscr{T})$-системы.

Теорема 6.1. Движение $\varphi(t) \in \Phi(A, \Phi, \mathscr{T})$-системы с запаздыванием устойчиво в смысле Ляпунова в том и только том случае, если существует положсительно определенная обобщенная функиия Ляпунова $V_{\tau}: A \times A \rightarrow \mathbb{R}^{+}$ со следующими свойствами:

1) для заданного числа $\varepsilon>0$ существует число $\delta(\varepsilon, \tau)>0$ такое, что

$$
p_{\Pi}\left(\varphi^{\prime}, \psi^{\prime}\right)<\delta \Rightarrow V_{\tau}(\varphi, \psi)<\varepsilon \quad \forall \psi \in \Phi
$$

2) для заданного числа $\eta>0$ существует число $\mu(\eta, \tau)>0$ такое, что

$$
V_{\tau}(\varphi, \psi)<\mu \Rightarrow p_{U}\left(\varphi^{\prime}, \psi^{\prime}\right)<\eta \quad \forall \psi \in \Phi \text {. }
$$

ДокАЗАтЕЛьство. Пусть существует обобщенная функция Ляпунова $V_{\tau}: A \times$ $A \rightarrow \mathbb{R}^{+}$, обладаюшая свойствами (6.1) и (6.2), и пусть числа $\varepsilon>0$ и $\tau \in \mathbb{R}^{+}$ заданы. При $\varepsilon=\eta$ подберем $\delta(\varepsilon, \tau)$ так, чтобы имело место: $\varphi\left(\varphi^{\prime}, \tau, t\right)=\varphi^{\prime}(t-\tau)$ при $t \in(\alpha+\tau, \tau]$. Поэтому движение $\varphi(t)$ устойчиво в смысле Ляпунова. Обратно, пусть движение $\varphi(t)$ устойчиво в смысле Ляпунова. Тогда для каждого $\varepsilon>0$ существует $\delta(\varepsilon, \tau)>0$ такое, что имеет место (2.16). Определим

$$
V_{\tau, t}(\varphi, \psi):=\sup \{p(\varphi(s), \psi(s)): s[\tau, t]\} .
$$

Тогда условие (6.1) следует из условия (2.19), а условие (6.2) справедливо при $\mu=\eta$. Теорема доказана. 
Теорема 6.2. Движение $\varphi(t) \in \Phi(A, \Phi, \mathscr{T})$-системы с запаздыванием асимптотически устойчиво в смысле Ляпунова в том и только том случае, если существует положительно определенная обобщенная функция Ляпунова $V_{\tau}: A \times A \rightarrow \mathbb{R}^{+}$со следующими свойствами:

1) имеют место условия (6.1) и (6.2) теоремь 6.1;

2) выполнено условие

$$
\exists h(\tau)>0 \quad \forall \psi^{\prime} \in B_{\Pi}\left(\varphi^{\prime}, h\right) \quad V_{s}(\varphi, \psi) \rightarrow 0, \quad s \rightarrow+\infty .
$$

ДоказАТЕльство. Пусть сушествует обобшенная функция Ляпунова $V_{\tau}$ со свойствами (6.1), (6.2), (6.4). Тогда по теореме 6.1 движение $\varphi(t) \in \Phi$ устойчиво в смысле Ляпунова. Предположим, что при условиях теоремы 6.2 движение $\varphi(t)$ не является асимптотически устойчивым. Тогда для каждого $h>0$ существует предыстория $\psi^{\prime} \in B_{\Pi}\left(\varphi^{\prime}, h\right)$ такая, что для одной истории $\psi(t)$ функция $p(\varphi(t), \psi(t))$ не стремится при $t \rightarrow+\infty$ к нулю. Это означает, что эта функция превосходит некоторое число $\eta$ при сколь угодно больших $t$ и, следовательно, $p_{U}\left(\varphi^{\prime \prime}, \psi^{\prime \prime}\right)>\eta$ $\forall \tau \in \mathbb{R}^{+}$. Из условия (6.2) вытекает теперь, что имеется число $\mu>0$ такое, что справедливо неравенство $V_{\tau}(\varphi, \psi)>\mu \forall \tau \in \mathbb{R}^{+}$, которое противоречит (6.4). Поэтому движение $\varphi(t)$ асимптотически устойчиво в смысле Ляпунова. Обратно, пусть $\varphi(t)$ асимптотически устойчиво. Определим

$$
V_{\tau, t}(\varphi, \psi):=\sup \{p(\varphi(s), \psi(s)): s \in[\tau, t]\} .
$$

В силу ограниченности решения имеем

$$
\exists h(\tau)>0 \quad \forall \psi^{\prime} \in B_{\Pi}\left(\varphi^{\prime}, h\right) \quad p(\varphi(t), \psi(t)) \rightarrow 0, \quad t \rightarrow+\infty .
$$

Следовательно,

$$
V_{s}(\varphi, \psi)=\sup \{p(\varphi(t), \psi(t)): t \geqslant s\} \rightarrow 0, \quad s \rightarrow+\infty,
$$

и требование (6.4) выполнено. Выполнение требований (6.1) и (6.2) вытекает из доказательства теоремы 6.1. Теорема 6.2 доказана.

Имеют место следующие теоремы.

Теорема 6.3. Пусть задана $(A, \Phi, \mathscr{T})$-система без запаздывания. Движение $\varphi(t) \in \Phi$ устойчиво в смысле Ляпунова в том и только том случае, если существует обобщенная функиия Ляпунова $V_{a}: A \times A \rightarrow \mathbb{R}^{+}$со свойствами:

$$
\begin{aligned}
& \forall \varepsilon>0 \quad \exists \delta(\varepsilon, a)>0 \quad p(\varphi(a), \psi(a))<\delta \Rightarrow V_{a}(\varphi, \psi)<\varepsilon, \\
& \forall \eta \quad \exists \mu(\eta, a)>0 \quad V_{a}(\varphi, \psi)<\mu \Rightarrow p(\varphi, \psi)<\eta \quad \forall \psi \in \Phi .
\end{aligned}
$$

Теорема 6.4. Пусть задана $(A, \Phi, \mathscr{T})$-система без запаздывания. Движение $\varphi(t) \in \Phi$ асимптотически устойчиво в смысле Ляпунова в том и только том случае, если существует обобщенная функиия Ляпунова $V_{a}: A \times A \rightarrow \mathbb{R}^{+}$ со свойствами (6.7), (6.8) и свойством

$$
\forall h(a)>0 \quad p(\varphi(a), \psi(a))<h \Rightarrow V_{a}(\varphi(t)) \rightarrow 0, \quad a \rightarrow+\infty .
$$

Теоремы 6.3 и 6.4 являются очевидньми следствиями теорем 6.1 и 6.2 соответственно, так как $(A, \Phi, \mathscr{T})$-система рассматривается в случае, когда предыстории движений совпадают с их начальными значениями.

Теоремы Зубова $[14 ;$ гл.4, § 2] и теоремы Мовчана [16] являются частными случаями теорем 6.3 и 6.4 . 


\section{$\S 7$. Устойчивость в $\Sigma$-системе и обобщенные функции Ляпунова относительно квазифильтров}

В этом параграфе рассмотрена унификация понятия устойчивости множества для общей системы “вход-выход” с заданным предпорядком. Результаты об устойчивости носят весьма общий характер. Все используемые здесь объекты систем описываются как абстрактные множества, и вводится лишш одно вспомогательное упорядоченное множество для того, чтобы определить понятие обобщенной функции Ляпунова. Вся информация о системе сосредоточена в определении некоторого предпорядка. Ограничившись только структурой предпорядка, можно тем не менее доказать основные теоремы метода обобщенных функций Ляпунова и получить критерии устойчивости в терминах обобщенных функций Ляпунова. Эти результаты могут быть перенесены и на общие системы с более богатой математической структурой фазового пространства, например на системы, определенные на множествах с произвольной топологией, с топологией равномерной сходимости, на метрических пространствах, на банаховых пространствах.

7.1. Устойчивость отображения относительно двух квазифильтров. Пусть $A$ - произвольное абстрактное множество и $S(A)$ - совокупность всех подмножеств множества $A$. Любая заданная совокупность $\mathscr{E}_{A} \subset S(A)$ назьвается квазифильтром на множестве $A$. Окрестностью $N(a)$ точки $a \in A$ в изучаемой общей системе "вход-выход" назовем множество

$$
N(a):=\left\{\alpha: \alpha \in \mathscr{E}_{A} \wedge a \in \alpha\right\}
$$

ОПРЕДЕЛЕНИЕ 7.1 (ключевое определение). Рассмотрим отображение

$$
F: P \rightarrow Q
$$

множества $P$ в множество $Q$. Пусть $\mathscr{E}_{P}$ и $\mathscr{E}_{Q}-$ некоторые квазифильтры на множествах $Р$ и $Q$ соответственно. Пусть $(\widehat{p}, \widehat{q}) \in P \times Q, \widehat{q}=F(\widehat{p})$. Пара $(\widehat{p}, \widehat{q})$ называется устойчивой относительно двух квазифильтров $\mathscr{E}_{P}$ и $\mathscr{E}_{Q}$, если

$$
\forall \alpha \in N(\widehat{q}) \quad \exists \beta \in N(\widehat{p}) \quad \forall p \quad p \in \beta \Rightarrow F(p) \in \alpha
$$

где $N(\widehat{q}) \subset E_{Q}, N(\widehat{p}) \subset E_{P}-$ квазифильтры точек $\widehat{q}$ и $\widehat{p}$ относительно $\mathscr{E}_{Q}$ и $\mathscr{E}_{P}$ соответственно.

Определение 7.1 названо нами ключевьлм, так как оно, как будет показано ниже, содержит многие определения различных видов устойчивости: определение структурной устойчивости, определение устойчивости реакции относительно двух квазифильтров, определение устойчивости состояния системы относительно квазифильтра и предпорядка, определение устойчивости состояния равновесия в смысле Ллпунова, определение орбитальной устойчивости множества, определение устойчивости изолированной траектории в смысле Немыцкого-Степанова, определение устойчивости изолированной траектории в смысле Пуассона, определение устойчивости изолированной траектории в смысле Якоби.

Конечно, имеются виды устойчивости, которые не охватываются определением 7.1, поскольку понятие устойчивости зависит как от вида системы, так и от того, каким образом вводится понятие окрестности. 
7.2. Структурная устойчивость $\Sigma$-системы. Рассмотрим отображение $F: P \rightarrow Q$. Произведем параметризацию общей системы $\Sigma \subset X \times Y$ с помощью множества $P$, а именно будем предполагать, что существует такое отображение $\Omega: \bar{\Sigma} \rightarrow Q$, что $\Omega(\Sigma)=q$, где $\Sigma \in \bar{\Sigma}$ содержит точку $q$ и $\bar{\Sigma}=\{\Sigma \subset X \times Y\}$. Будем также предполагать, что $R: P \rightarrow \bar{\Sigma}$, а отображение $F: P \rightarrow Q$ является композицией отображений $\Omega$ и $R$, т.е. $q=F(p)=\Omega(R(p))$.

Рассмотрим вырожденный случай, когда в качестве системы окрестностей точки $q \in Q$ принимается $N(q)=\{q\}$.

ОПРЕДЕЛЕнИЕ 7.2. Точка $\widehat{q} \in Q$ $\Sigma$-системы называется структурно устойчивой, если для любой точки $\widehat{p} \in P$, где $\widehat{q}=F(\widehat{p})$, сушествует такое $\beta \in N(\widehat{p})$, что для всех $p$ имеет место импликация

$$
p \in \beta \Rightarrow F(p)=\widehat{q}
$$

Очевидно, что определение 7.2 является частным случаем определения 7.1.

7.3. Устойчивость реакции. Понятие устойчивости реакции является частньм случаем определения 7.1. Пусть $P$ - начальный объект состояний $\Phi, \mathscr{E}$ - выходной объект $Y, F$ - начальная реакция $R_{0}$ системы для заданного входного воздействия $\widehat{x} \in X$. Пусть $\mathscr{E} \Phi$ и $\mathscr{E} Y-$ заданные квазифильтры на множествах $\Phi$ и $Y$ соответственно. Тогда определение 7.1 равносильно следуюшему определению.

ОПРеДЕЛЕнИЕ 7.3. Пусть $\widehat{x} \in X-$ входное воздействие. Реакция $\widehat{y}=R_{0}\left(\widehat{\varphi}_{0}, \widehat{x}\right)$ $\Sigma$-системы называется устойчивой относительно заданного входного воздействия $\widehat{x}$ и заданной пары $\left(\mathscr{E}_{\Phi}, \mathscr{E}_{Y}\right)$ квазифильтров $\mathscr{E}_{\Phi}$ и $\mathscr{E}_{Y}$, если

$$
\forall \alpha \in N(\widehat{y}) \quad \exists \beta \in N\left(\widehat{\varphi}_{0}\right) \quad \varphi_{0} \in \beta \Rightarrow R_{0}\left(\varphi_{0}, \widehat{x}\right) \in \alpha,
$$

где $N(\widehat{y})$ - квазифильтр точки $\widehat{y}$ и $N\left(\widehat{\varphi}_{0}\right)$ - квазифильтр точки $\widehat{\varphi}_{0}$.

Для динамической $\Sigma$-системы задача об устойчивости решается обычно на языке семейства функций перехода состояний. Если эта система допускает каноническое представление $(\bar{f}, \bar{\lambda})$, то указанный подход законен, так как выходная функция $\bar{\lambda}$ не будет влиять на устойчивость динамической системы. При подобном подходе единственная информация о $\Sigma$-системе, необходимая для анализа вопроса об устойчивости, заключена в последовательной смене состояний. Итак, вся необходимая информация об общей системе в этом случае может быть представлена посредством некоторого упорядочения $\Pi \subset \Phi \times \Phi$ пространства состояний.

Имеет место следуюшая

ЛЕмма 7.1. Пусть инвариантная по времени динамическая $\Sigma$-система $(\bar{f}, \bar{\lambda})$ обладает пространством состояний $\Phi$. Пусть $Z: T \rightarrow \Phi$ и для любого $x \in X$ инвариантная по времени $\Sigma$-система задает отночение $\Pi \subset \Phi \times \Phi$ makoe, чmo

$$
\left(\varphi, \varphi_{1}\right) \in \Pi \Leftrightarrow \exists x_{t t^{\prime}} \quad \varphi_{1}=f_{t t^{\prime}}\left(\varphi, x_{t t^{\prime}}\right) .
$$

Тогда справедливы соотношения

$$
\begin{gathered}
(\varphi, \varphi) \in \Pi \quad \forall \varphi \in \Phi, \\
\left(\varphi, \varphi_{1}\right) \in \Pi \wedge\left(\varphi_{1}, \varphi_{2}\right) \in \Pi \Rightarrow\left(\varphi, \varphi_{2}\right) \in \Pi \quad \forall\left(\varphi, \varphi_{1}, \varphi_{2}\right) \in \Phi .
\end{gathered}
$$


ДоказАТЕЛЬСтво. Свойство (7.6) вытекает из свойства $f_{t t}\left(\varphi, x_{t t}\right)=\varphi$, в то время как свойство (7.7) есть следствие инвариантности по времени и свойства полугруппы. В самом деле, пусть $\left(\varphi, \varphi_{1}\right) \in \Pi$ и $\left(\varphi_{1}, \varphi_{2}\right) \in \Pi$, т.е. пусть $\varphi_{1}=f_{t t^{\prime}}\left(\varphi, x_{t t^{\prime}}\right)$ и $\varphi_{2}=f_{s s^{\prime}}\left(\varphi_{1}, \widehat{x}_{s s^{\prime}}\right)$ для некоторых $x_{t t^{\prime}}$ и $\widehat{x}_{s s^{\prime}}$. Положим $t_{2}=\left(s_{1}-s\right)+t_{1}$. В силу инвариантности по времени $\Sigma$-системы множество моментов времени есть линейно упорядоченная абелева группа. Но тогда $\varphi_{2}=$ $f_{t_{1} t_{2}}\left(\varphi_{1}, F^{t_{1}-s}\left(\widehat{x}_{s s^{\prime}}\right)\right)$. Поскольку

$$
x_{t t_{2}}=x_{t t_{1}} \cdot F^{t_{1}-s}\left(\widehat{x}_{s s_{1}}\right) \in X_{t t_{2}}
$$

и

$$
\begin{aligned}
\varphi_{2}=f_{t_{1} t_{2}}\left(\varphi_{1}, F^{t_{1}-s}\left(\widehat{x}_{s s_{1}}\right)\right) & =f_{t_{1} t_{2}}\left(f_{t t_{1}}\left(\varphi, x_{t t_{1}}\right), F^{t_{1}-s}\left(\widehat{x}_{s s_{1}}\right)\right) \\
& =f_{t t^{\prime \prime}}\left(\varphi, x_{t t_{1}} \cdot F^{t_{1}-s}\left(\widehat{x}_{s s_{1}}\right)\right)
\end{aligned}
$$

то $\left(\varphi, \varphi_{2}\right) \in$ П. Лемма доказана.

В определении 7.1 примем $P=\Phi, Q=S(\Phi)$. Пусть функция $F: \Phi \rightarrow S(\Phi)$ обладает свойством

$$
F(\varphi)=\Pi(\varphi)=\left\{\varphi^{\prime}:\left(\varphi, \varphi^{\prime}\right) \in \Pi\right\} .
$$

Предположим также, что

$$
\mathscr{E}_{P}=\mathscr{E}_{Q}=\mathscr{E} \subset S(\Phi)
$$

Тогда определение 7.1 равносильно в силу леммы 7.1 следуюшему определению.

ОПРЕДЕЛЕНИЕ 7.4. Пусть $\mathscr{E}_{P}=\mathscr{E}_{Q}=\mathscr{E} \subset S(\Phi)$ и П $\subset \Phi \times \Phi$. Одноточечное множество $\{\varphi\} \in \Phi$ назьвается устойчивым относительно квазифильтра $\mathscr{E}$, если

$$
\forall \alpha \in N(\Pi(\varphi)) \quad \exists \beta \in N(\varphi) \quad \Pi(\beta) \subset \alpha,
$$

где $\Pi(\beta):=\bigcup_{\varphi \in \beta} \Pi(\varphi), N(\Pi(\varphi)):=\{\alpha: \alpha \in \mathscr{E} \wedge \Pi(\varphi) \subset \alpha\}$.

В определении $7.4 \Sigma$-система фигурирует лиш в виде порождаемого ею отношения предпорядка П, обладаюшего свойствами (7.5) и (7.6). Определение 7.4 есть определение устойчивости предпорядка. Важно отметить, что отношение П определено семейством функций перехода состояний некоторой системы.

7.4. Устойчивость состояния равновесия классической динамической $\left(\mathbb{R}^{n}, f, \mathbb{R}\right)$-системы. Рассмотрим $\left(\mathbb{R}^{n}, f, \mathbb{R}\right)$-систему, топология которой определена какой-нибудь нормой пространства $\mathbb{R}^{n}$. Квазифильтр $\mathscr{E}$ соответствует этой топологии. Пусть

$$
f_{0 t}: \Phi \rightarrow \Phi, \quad f_{0 t}(\widehat{\varphi})=\widehat{\varphi} \quad \forall t .
$$

Тогда $\widehat{\varphi} \in \Phi$ - равновесное состояние рассматриваемой системы.

Определение 7.4 равносильно для одноточечного множества следуюшему определению. 
ОПРЕДЕЛЕНИЕ 7.5. Одноточечное множество $\{\varphi\} \in \Phi\left(\mathbb{R}^{n}, f, \mathbb{R}\right)$-системы называется устойчивым в смысле Ляпунова, если

$$
\forall \varepsilon>0 \quad \exists \delta>0 \quad\|\varphi-\widehat{\varphi}\|<\delta \Rightarrow\left\|f_{0 t}(\varphi)-\widehat{\varphi}\right\|<\varepsilon \quad \forall t \in \mathbb{R}^{+} .
$$

Определение 7.5 есть определение устойчивости в смысле Ляпунова состояния равновесия $\varphi \in \Phi$. Поэтому определение 7.3 есть обобщение понятия устойчивости в смысле Ляпунова.

Определение устойчивости одноточечного множества распространим на произвольное множество $M \subset \Phi$.

ОПРЕДЕЛЕНИЕ 7.6. Множество $M \subset \Phi$ называется орбитально устойчивым. относительно квазифильтра $\mathscr{E}$, если

$$
\forall \alpha \in N(\Pi(M)) \quad \exists \beta \in N(M) \quad \Pi(\beta) \subset \alpha .
$$

7.5. Устойчивость отдельной орбиты. Устойчивость некоторого заданного элемента П $(\widehat{\varphi})$ была ранее рассмотрена относительно других элементов $(\beta)$. Определим устойчивость другого типа, когда устойчивость множества $\Pi(\widehat{\varphi})$ рассматривается относительно самого множества П $(\widehat{\varphi})$. Примером такой устойчивости является устойчивость в смысле Пуассона [20].

ОПРЕДЕЛЕНИЕ 7.7 . Пусть $(A, f, \mathbb{R})$-система с заданным входным воздействием $\widehat{x}$ описывается уравнением $\widehat{z}(t, \varphi)=f_{0 t}\left(\varphi, \widehat{x}^{t}\right)$. Точка $\varphi$ или орбита $C(\varphi): \mathbb{R}^{+} \rightarrow \Phi$ решения $\widehat{z}(t, \varphi)$ называется устойчивой в смысле Пуассона, если

$$
\exists \widehat{t} \forall \alpha \in N(\widehat{z}(\widehat{t}, \varphi)), \forall t \quad \exists t^{\prime} \quad t \leqslant t^{\prime} \Rightarrow \widehat{z}(\widehat{t}, \varphi) \in \alpha .
$$

Другими словами, точка $\varphi$ устойчива в смысле Пуассона, если найдутся сколь угодно большие значения $t$, при которых точка оказывается в любой окрестности своего начального положения [20].

ОПредЕлЕниЕ 7.8. Пусть задана $\left(\mathbb{R}^{n}, f, \mathbb{R}\right)$-система с топологией, определяемой нормой пространства $\mathbb{R}^{n}$, и существует только одно входное воздействие $\widehat{x}$. Рассмотрим решение $\widehat{z}(t)=f_{0 t}(\varphi, \widehat{x})$. Орбита $C: \mathbb{R}^{+} \rightarrow \Phi$ решения $\widehat{z}(t)$ называется устойчивой в смысле Немыцкого-Степанова, если

$$
\forall \varepsilon \quad \exists \widehat{t} \forall t, \forall t_{1} \quad \widehat{t} \leqslant t \wedge \widehat{t} \leqslant t_{1} \Rightarrow\left\|\widehat{z}(t)-\widehat{z}\left(t_{1}\right)\right\|<\varepsilon .
$$

Определение 7.8 является определением устойчивости изолированной орбиты $C$ относительно самой себя. В обшем случае понятия устойчивости орбиты в смысле Немыцкого-Степанова и устойчивости орбиты в смысле Пуассона являются различньми независимыми понятиями [20].

7.6. Ключевой критерий орбитальной устойчивости множества $\Sigma$-системы. Установим критерий орбитальной устойчивости множества общей временной $\Sigma$-системы, который без изменений переносится на системы с более богатой математической структурой. 
ОПРЕДЕЛЕНИЕ 7.9. Пусть $Г$ - частично упорядоченное множество, $\Gamma^{+}-$некоторое его фиксированное подмножество и $\Pi \subset \Phi \times \Phi$. Функция $V: \Phi \rightarrow \Gamma$, обладающая свойствами

$$
\begin{gathered}
\forall \varphi, \forall \varphi_{1} \quad\left(\varphi, \varphi_{1}\right) \in \Pi \Rightarrow V(\varphi) \geqslant V\left(\varphi_{1}\right), \\
\forall n \quad \exists \alpha \quad \forall \varphi \quad n \in \Gamma^{+} \wedge M \subset \alpha \wedge \varphi \in \alpha \Rightarrow V(\varphi) \leqslant n, \\
\forall \alpha \quad \exists n \quad \forall \varphi \quad n \in \Gamma^{+} \wedge \Pi(M) \subset \alpha \wedge V(\varphi) \leqslant n \Rightarrow \varphi \in \alpha,
\end{gathered}
$$

называется обобщенной функиией Ляпунова $\Sigma$-системы для множества $M \subset \Phi$.

Определение 7.9 охватывает как частные случаи определения обобщенных функций Ляпунова, данные в $\S \S 2-6$.

Имеет место следующая

ТЕОРема 7.1 (ключевой критерий). Пусть

1) задана $\Sigma$-система,

2) $\Phi$ - некоторое абстрактное множество,

3) П - отношение предпорядка в $\Phi$,

4) $\mathscr{E}$ - произвольный квазифильтр на множестве $\Phi$.

Для того чтобы множсество $M \subset \Phi$ было орбитально устойчивым относительно квазифильтра $\mathscr{E}$, необходимо и достаточно, чтобы существовала обобщенная функция Ляпунова $V: \Phi \rightarrow \Gamma$, обладающая свойствами (7.13)-(7.15).

ДокАЗАТЕЛЬСтво. Необходимость. Пусть множество $M \subset \Phi$ орбитально устойчиво относительно квазифильтра $\mathscr{E}$. Покажем, что существует обобщенная функция Ляпунова $V: \Phi \rightarrow \Gamma$ со свойствами (7.13)-(7.15). Выберем $\Gamma=D, \widehat{\Phi}=$ $\Pi(M)$. Определим

$$
V(\varphi)=\sup _{\varphi_{1} \in \Pi(\varphi)}\left(\widehat{\Phi}, \varphi_{1}\right)
$$

Так как П транзитивно, то условие (7.13) выполнено. Выберем $n \in \Gamma^{+}=D_{\widehat{\varphi}}$. Так как $\widehat{\Phi} \subset S(\widehat{\Phi}, n) \in N(\widehat{\Phi})$, то в силу предложения 2.6 и свойства орбитальной устойчивости $M$ сушествует $\alpha \in N(M)$ такое, что

$$
\forall \varphi, \forall \varphi_{1} \quad \varphi \in \alpha \wedge\left(\varphi, \varphi_{1}\right) \in \Pi \Rightarrow R\left(\widehat{\Phi}, \varphi_{1}\right) \leqslant n .
$$

Следовательно, условие (7.14) вьполнено. Выберем, далее, $\alpha \in N(\Phi)$. Тогда в силу предложения 2.6 сушествует $n \in \Gamma^{+}$такое, что $\widehat{\Phi} \subset S(\widehat{\Phi}, n) \subset \alpha$. Если $V(\varphi) \leqslant n$, то будем иметь

$$
n \geqslant V(\varphi)=\sup _{\varphi_{1} \in \Pi(\varphi)} R\left(\widehat{\Phi}, \varphi_{1}\right) \geqslant R(\widehat{\Phi}, \varphi) .
$$

Следовательно, условие (7.15) также выполнено.

Достаточность. Пусть выполнены условия (7.13)-(7.15). Выберем какое-нибудь $\alpha \in N(\Pi(M))$. Из условия (7.15) следует, что для некоторого $\widehat{n} \in \Gamma^{+}$имеет место $\{c: V(c) \leqslant \widehat{n}\} \subset \alpha$. Из (7.13) и (7.14) соответственно вытекает, что

$$
V(\varphi)<\widehat{n}, \quad\left(\varphi, \varphi_{1}\right) \in \Pi \Rightarrow V\left(\varphi_{1}\right) \leqslant \widehat{n} .
$$

Следовательно, $\Pi(\beta) \subset\{\varphi: V(\varphi) \leqslant \widehat{n}\} \subset \alpha$ и, значит, множество $M$ орбитально устойчиво относительно квазифильтра $\mathscr{E}$. Теорема доказана. 
ОПРЕДЕЛЕНИЕ 7.10. Функция $V: \Phi \rightarrow \Gamma$ называется обобщенной функиией Ляпунова $\Sigma$-системы для инвариантного множества $M \subset \Phi$, если

$$
\begin{gathered}
\forall \varphi, \forall \varphi_{1} \quad\left(\varphi, \varphi_{1}\right) \in \Pi \Rightarrow V(\varphi) \geqslant V\left(\varphi_{1}\right), \\
\forall n \quad \exists \alpha \quad \forall \varphi \quad n \in \Gamma^{+} \wedge M \subset \alpha \wedge \varphi \in \alpha \Rightarrow V(\varphi) \leqslant n, \\
\forall \alpha \quad \exists n \quad \forall \varphi \quad n \in \Gamma^{+} \wedge M \subset \alpha \wedge V(\varphi) \leqslant n \Rightarrow \varphi \in \alpha .
\end{gathered}
$$

Различие между определениями 7.9 и 7.10 обобщенной функции Ляпунова общей $\Sigma$-системы для неинвариантного и инвариантного множеств заключается в том, что в определении 7.9 множество $\alpha$ содержит П $(M)$, а в определении 7.10 множество $\alpha$ содержит лишь множество $M$. Оба определения относятся как к ограниченным, так и неограниченньм множествам, и это дает возможность унифицировать определения для большого числа понятий обобщенной функции Ляпунова.

7.7. Критерий орбитальной устойчивости множества в топологическом фазовом пространстве. Пусть $\Phi$ - топологическое пространство с топологией $\mathscr{E}$. Имеет место следуюшая

Tеорема 7.2. Пусть

1) задана $\Sigma$-система, для которой $\Phi$ - топологическое пространство $c$ топологией $\mathscr{E}$,

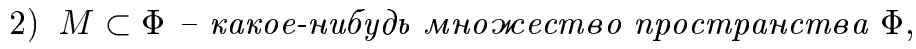

3) П - некоторое отношение предпорядка в $\Phi$.

Мнохсество $М$ орбитально устойчиво относительно квазифильтра $\mathscr{E}$ в том и только том случае, если существует обобщенная функиия Ляпунова $V: \Phi \rightarrow \Gamma$, әде Г - частично упорядоченное множество из $\Phi$.

ДокАЗАТЕЛЬСтво. Легко проверить, что ключевой критерий сохраняет свою силу и тогда, когда $\mathscr{E}$ определяет топологию, т.е. когда выполнены следуюшие условия:

a) $\Phi \in \mathscr{E}$ и $\varnothing \in \mathscr{E}$;

б) объединение любого числа элементов из $\mathscr{E}$ также принадлежит $\mathscr{E}$;

в) пересечение любого конечного числа элементов из $\mathscr{E}$ также является элементом $\mathscr{E}$.

Очевидно, что введение дополнительно топологической структуры не приводит к нарушению условий ключевого критерия. Теорема доказана.

7.8. Критерий орбитальной устойчивости компактного множества в метрическом фазовом пространстве. Пусть $\Phi$ - метрическое фазовое пространство с метрикой $R: \Phi \times \Phi \rightarrow \mathbb{R}^{+}$и топологией $\mathscr{E}$, порожденной этой метрикой. Для множества $M \subset \Phi$ определим

$$
R(M, \varphi):=\inf \left\{R\left(\varphi_{1}, \varphi\right), \varphi_{1} \in M\right\}, \quad B(M, \varepsilon):=\{\varphi: R(M, \varphi)<\varepsilon\} .
$$

Легко проверить, что при $D_{\varphi_{1}}=\mathbb{R}^{+}$справедливы соотношения

$$
\begin{gathered}
R(M, \varphi) \geqslant 0, \\
R(M, \varphi)=0, \text { если } \varphi \in M, \\
\forall \psi \quad \psi \in D_{\varphi} \Rightarrow B(M, \psi) \in N(M),
\end{gathered}
$$

где $N(M)$ - совокупность всех открытых множеств, содержащая и $M$. 
Установим, что для компактного множества $M$ имеет место также следующее свойство:

$$
\forall \alpha \in N(M) \quad \exists \psi \in D_{\varphi} \quad M \subset B(M, \psi) \subset \alpha .
$$

Действительно, если свойство $\left(7.18^{\prime}\right)$ несправедливо, то для любого целого числа $n$ существует $\varphi_{n}$ такое, что

$$
\begin{gathered}
\varphi_{n} \in B\left(M, \frac{1}{n}\right) \cap(\Phi \backslash \alpha), \\
\varphi_{n} \in B\left(M, \frac{1}{n}\right) .
\end{gathered}
$$

Из (7.20) следует, что

$$
\exists \widehat{\varphi}_{n} \in M \quad R\left(\varphi_{n}, \widehat{\varphi}_{n}\right)<\frac{2}{n} .
$$

В силу компактности множества $M$ последовательность $\widehat{\varphi}_{n}$ имеет предельную точку $\widehat{\varphi}_{0} \in M$. Предположим, что $\widehat{\varphi}_{n}$ сходится к $\widehat{\varphi}_{0}$ при $n \rightarrow \infty$. Тогда

$$
R\left(c_{n}, \widehat{c}_{0}\right) \leqslant R\left(c_{n}, \widehat{c}_{n}\right)+R\left(\widehat{c}_{n}, \widehat{c}_{0}\right) \rightarrow 0, \quad n \rightarrow \infty .
$$

Но (7.21) противоречит тому, что $\varphi_{n} \in M \backslash \alpha$. Таким образом, $\left(7.18^{\prime}\right)$ установлено.

Из доказательства теоремы 7.1 вытекает, что существование обобщенной функции Ляпунова, обеспечивающей орбитальную устойчивость множества $M^{*}=\Pi^{-1}(M)$, определяется условиями (7.18) или $\left(7.18^{\prime}\right)$ и что обобшенная функция Ляпунова имеет вид

$$
V(\varphi)=\sup \left\{R\left(\Pi(M), \varphi_{1}\right), \varphi_{1} \in \Pi(\varphi)\right\} .
$$

$\mathrm{B}$ частности, для инвариантного множества $M$ обобшенная функция Ляпунова определяется соотношением

$$
V(\varphi)=\sup \left\{R\left(M, \varphi_{1}\right), \varphi_{1} \in \Pi(\varphi)\right\} .
$$

Имеет место следующая

Tеорема 7.3. Пусть

1) задана $\Sigma$-система,

2) $\Gamma^{+}=\mathbb{R}^{+}$,

3) $(\Phi, \mathscr{E})$ - метрическое пространство,

4) $K \subset \Phi$ - компактное мнозсество пространства $\Phi$,

5) П - некоторое отношение предпорядка в $\Phi$.

Множество К орбитально устойчиво относительно предпорядка $(\mathscr{E}, \Pi)$ в том и только том случае, если существует обобщенная функиия Ляпунова $V: \Phi \rightarrow \mathbb{R}^{+}$.

ДокАЗАТЕЛЬСтво. Достаточность условий очевидна. Установим необходимость условий. Предположим, что справедливо условие $\left(7.18^{\prime}\right)$. Из существования числа $k, 0<k<n$, такого, что $K \subset B(K, k) \subset B(K, n) \subset \alpha$, следует, что $k \geqslant R(K, \varphi)$. Следовательно, $\varphi \in B(K, n) \subset \alpha$ при $n \geqslant R(K, \varphi)$. Теорема доказана. 


\section{Список литературы}

1. Ляпунов А. М. Общая задача об устойчивости движения. Харьков: Изд-во Харьковского матем. об-ва, 1892.

2. Пуанкаре А. Избранные труды. Т. 1, 2. М.: Наука, 1971, 1972.

3. Четаев Н. Г. Устойчивость движения. М.: ГИТТЛ, 1955.

4. Румянцев B. В., Озиранер А. С. Устойчивость и стабилизация движения по отношению к части переменных. М.: Наука, 1987.

5. Воротников В.И., Румянцев В.В. Устойчивость и управление по части координат фазового вектора динамических систем: теория, методы и приложения. М.: Научный мир, 2001.

6. Руш Н., Абетс П., Лапуа М. Прямой метод Ляпунова в теории устойчивости. М.: Мир, 1980.

7. Бабин А. В., Вишик М.И. Аттракторы эволюционных уравнений. М.: Наука, 1989.

8. Шестаков A. A. Обобщенный прямой метод Ляпунова для систем с распределенными параметрами. М.: Наука, 1990.

9. Kato J., Martynyuk A. A., Shestakov A. A. Stability of motion of nonautonomous systems (method of limiting equations). Amsterdam: Gordon and Breach, 1996.

10. Румянцев В. В. О развитии исследований в СССР по теории устойчивости движения // Дифференц. уравнения. 1983. Т. 19. № 5. С. 739-776.

11. Румянцев B. В. Об устойчивости движения по отношению к части переменных // Вестн. МГУ. Сер. матем., мех., физ., астрон., хим. 1957. №4. С. 9-16.

12. Персидский К. П. Избранные труды. Т. 1, 2. Алма-Ата: Наука, 1973.

13. Красовский H. Н. Некоторые задачи теории устойчивости движения. М.: Физматгиз, 1959.

14. Зубов В. И. Методы Ляпунова и их применение. Л.: Изд-во ЛГУ, 1957.

15. Матросов В. М. Метод векторных функций Ляпунова: анализ динамических свойств нелинейных систем. М.: Физматлит, 2001.

16. Мовчан A. А. Устойчивость процессов по двум метрикам // Прикл. матем. и мех. 1960. T. 24. №6. C. $988-1001$.

17. Барбашин E. A. К теории обобщенных динамических систем // Ученые записки МГУ. 1948. T. 135. № 2. C. 110-133.

18. Bushaw D. D. A stability criterion for general systems // J. Math. Systems Theory. 1967. V. 1. № 1. P. 79-88.

19. Немыцкий В.В. К теории орбит общих динамических систем // Матем. сб. 1948. T. 23(65). № 2. C. 161-186.

20. Немыцкий B. B., Степанов В. В. Качественная теория дифференциальных уравнений. М.-Л.: Гостехиздат, 1949.

21. Yoshii S. General stability of sets // M.S. thesis. Cleveland: Case Western Reserve Univ., 1971.

22. Сиразетдинов Т. К. Устойчивость систем с распределенными параметрами. Новосибирск: Наука, 1987.

23. Малышев Ю. В. Методы обобщенных функций Ляпунова. // Дисс. . . докт. физ.-матем. наук. Свердловск, 1991.

24. Меренков Ю. Н. Устойчивоподобные свойства дифференциальных включений, нечетких и стохастических дифференциальных уравнений. М.: Изд-во РУДН, 2000.

25. Дружинина O.B., Шестаков A.A. О связи устойчивости по Ляпунову с устойчивостью по Пуанкаре для движений нелинейной стационарной динамической системы // Докл. РАН. 1996. Т. 351. № 1. С. 48-51.

26. Дружинина O. В., Шестаков $A . A$. О понятиях орбиталшной устойчивости и фазовой устойчивости движений динамической системы // Докл. РАН. 1997. Т. 355. №3. C. 339-341.

27. Дружинина О.В., ШШестаков А. А. О неустойчивости состояния равновесия по первому приближению стационарного нелинейного уравнения в гильбертовом пространстве // Дифференц. уравнения. 1999. Т. 35. №6. С. 840.

28. Дружинина О. В., Шестаков A. A. О расширении понятия орбитальной устойчивости траекторий динамической системы // Докл. РАН. 2001. Т. 377. № 5. С. 621-625. 
29. Биркгоф Джс. Динамические системы. М.: Гостехиздат, 1941.

30. Bhatia N. P., Szegö G. P. Stability theory of dynamical systems. Berlin: Springer-Verlag, 1970 .

31. Seibert P. A unified theory of Lyapunov stability // Funkcial. Ekvac. 1972. V. 15. №3. P. 139-147.

32. Gilbert I. E., Knops R. T. Stability of general systems // Arch. Ration. Mech. Anal. 1967. V. 25. № 4. P. 271-297.

33. Шестаков A. А., Меренков Ю. Н. Об определениях и условиях устойчивости по Ляпунову для абстрактных динамических процессов // Сб. научн. трудов. Т. 140. М.: ВЗИИТ МПС, 1987. С. 40-50.

34. Месарович М., Такахара $Я$. Общая теория систем: математические основы. М.: Мир, 1978.

35. Ura T. On the flow outside a closed invariant set: stability, relative stability and saddle sets // Contrib. Differential Equations. 1964. V. 3. № 3. P. 249-294.

36. Auslander J., Seibert P. Prolongations and stability in dynamical systems // Ann. Inst. Fourier (Grenoble). 1964. V. 14. P. 237-267.

37. Моисеев Н. Д. Очерки развития теории устойчивости. М.-Л.: ГИТТЛ, 1949.

38. Демидович Б. П. Лекции по математической теории устойчивости. М.: Наука, 1967.

39. Auslander J. Filter stability in dynamical systems // SIAM J. Math. Anal. 1977. V. 8. № 4. P. 573-579.

40. Massera J. L. Contributions to stability theory // Ann. of Math. (2). 1956. V. 64. P. 182-206. 